Comment. Math. Helv. 75 (2000) 284-318

(C) 2000 Birkhäuser Verlag, Basel

$0010-2571 / 00 / 020284-35 \$ 1.50+0.20 / 0$

Commentarii Mathematici Helvetici

\title{
Oscillation, spiralement, tourbillonnement
}

\author{
F. Cano, R. Moussu and F. Sanz
}

À René Thom

Résumé. La dynamique oscillante d'un champ de vecteurs analytique en dimension trois s'organise autour d'un nombre fini d'axes de tourbillonnement lorsqu'elle ne se délocalise pas par des éclatements de point.

Abstract. The oscillating dynamics of an analytic vector field in dimension three is organized around a finite number of twister axis when it is localisable by point blowing-ups.

Mathematics Subject Classification (2000). 34Cxxm, 32B20.

\section{Introduction}

Soit $X$ un champ vecteurs analytique sur une variété $M$ et soit $t \mapsto \gamma(t)$ une courbe intégrale de $X$ dont l'ensemble $\omega$-limite $\omega(\gamma)$ est un point singulier $p$ de $X$. Dans ce travail nous étudions la question suivante: comment, d'un point de vue analytique, $\gamma$ peut-elle tendre vers $p$ ? C'est une question classique déjà étudiée à la fin du $19^{\text {ème }}$ siècle par Poincaré $[\mathrm{Po}]$ et Lyapunov [Ly]. En dimension 2, leurs travaux apportent une réponse satisfaisante à cette question: $\gamma$ possède une tangente en $p$ ou $\gamma$ spirale autour de $p$ et alors $p$ est un foyer monodromique. Pour décrire le comportement analytique de $\gamma$ en dimension $\geq 2$ nous utiliserons les concepts d'oscillation, de tangentes itérées et de spiralement axial en dimension 3. Donnons brièvement leur définition.

La courbe $\gamma:[0, \infty[\longrightarrow M$ est non oscillante si toute hypersurface analytique qui ne la contient pas la coupe un nombre fini de fois seulement. Soit $\pi_{1}: M_{1} \rightarrow M$ l'éclatement de $M$ de centre $p$. La courbe $\gamma$ possède (par définition) une tangente en $p$ si son relevé $\gamma_{1}=\pi_{1}^{-1} \circ \gamma$ possède un unique point $\omega$-limite $p_{1}=\omega\left(\gamma_{1}\right)$. S'il existe une suite d'éclatements

$$
M=M_{0} \stackrel{\pi_{1}}{\longleftarrow} M_{1} \stackrel{\pi_{2}}{\longleftarrow} M_{2} \cdots \longleftarrow M_{n-1} \stackrel{\pi_{n}}{\longleftarrow} M_{n} \cdots
$$

de centres respectifs $p=p_{0}, p_{1}, \ldots, p_{n-1}, \ldots$ tels que, pour tout $n$, le relevé $\gamma_{n}=$ $\pi_{n}^{-1} \circ \gamma_{n-1}$ possède un unique point $\omega$-limite $p_{n}=\omega\left(\gamma_{n}\right)$. On dit alors que $\gamma$ possède des tangentes itérées en $p$ et on écrit $T I(\gamma)=\left\{p_{n}\right\}$. On voit que si $\gamma$ est 
non oscillante alors $T I(\gamma)$ existe. La réciproque est vraie en dimension 2. Cette dichotomie osciller-tangentes itérées est propre à la dimension 2. En dimension 3, il faut la remplacer par le spiralement axial comme le montre le théorème suivant.

Théorème 1. Si TI $(\gamma)$ existe et si $\gamma$ est oscillante alors $\gamma$ spirale autour d'un axe analytique $\Gamma$ invariant par $X$.

La définition générale du spiralement axial étant un peu technique (voir 1.4), nous allons seulement en donner une propriété caractéristique lorsque $\Gamma$ est lisse: $\gamma$ spirale autour de $\Gamma$ lisse s'il existe des coordonnées analytiques $(x, y, z)$ en $p$ telles que $\Gamma=\{x=y=0, z>0\}$ et telles que si $\gamma(t)=(x(t), y(t), z(t))$ la courbe $t \mapsto$ $(x(t), y(t))$ spirale autour de 0 dans le plan réel et $\lim _{t \rightarrow \infty}\left(x^{2}(t)+y^{2}(t)\right) z(t)^{-n}=0$ pour tout $n$. L'hypothèse $\Gamma$ lisse n'est pas essentielle. En effet par une suite finie d'éclatements ponctuels on se ramène à ce cas.

Nous dirons qu'une demi-courbe analytique (ouverte) $\Gamma$ est un axe de tourbillonnement de $X$ s'il existe un voisinage semi analytique $V$ de $\Gamma$, positivement invariant par $X$ tel que $\Gamma$ soit un axe de spiralement pour toute courbe intégrale de $X$ issue d'un point de $V$. Il est dit non dégénéré si $\Gamma$ n'est pas contenu dans l'ensemble Sing $X$ des points fixes de $X$. Ce concept doit être rapproché de celui de foyer monodromique en dimension 2 comme le montre le théorème suivant.

Théorème 2. Un axe de spiralement non dégénéré $\Gamma$ pour $\gamma$ est un axe de tourbillonnement de $X$.

Ces axes sont des "centres organisateurs" de la dynamique de $X$ au sens de R. Thom. Ils possèdent la propriété de finitude que l'on exige en général de tels objets.

Corollaire. Tout point $p$ de $M$ possède un voisinage qui ne contient qu'un nombre fini d'axes de tourbillonnemment non dégénérés.

La motivation initiale de ce travail était l'étude de la dynamique d'un champ de gradient, $X=\nabla f$, où $f$ est une fonction analytique. S. Lojasiewicz a montré [Lo1] qu'une courbe intégrale $\gamma$ de $\nabla f$ a au plus un point $\omega$-limite $\omega(\gamma)=p$ et R. Thom $\left[\mathrm{Th}_{1}\right]$ a proposé la conjecture du gradient: $\gamma$ possède une tangente en $p$. Cette conjecture ${ }^{1}$ est vraie en dimension 2 et dans certains cas en dimension plus grande $[\mathrm{Hu}]$. On peut aussi proposer la conjecture forte du gradient: $\gamma$ n'est pas oscillante. Cette conjecture est encore vraie en dimension 2. Elle l'est aussi en dimension 3 lorsque la hessienne de $f$ en $p$ n'est pas nulle [Sa]. De plus, on peut montrer qu'un champ de gradient ne possède pas d'axe de tourbillonnement non dégénéré lisse [Fo-Sa]. Signalons enfin que dans un travail récent [Br], M. Brunella montre que tout champ de vecteurs analytique en dimension trois, à singularité

\footnotetext{
1 Récemment K. Kurdyka, T. Mostowski et A. Parusiński ont prouvé cette conjecture.
} 
isolée, possède une courbe intégrale ayant la propriété des tangentes itérées. Ainsi, les deux théorèmes précédents s'appliquent à ces champs de vecteurs.

Ce travail comporte deux parties. Dans la première partie, on étudie tout d'abord les relations entre les concepts oscillation, tangentes itérées et spiralement pour une courbe analytique quelconque $\gamma:\left[0, \infty\left[\rightarrow M\right.\right.$ telle que $\lim _{t \rightarrow \infty} \gamma(t)=p$. Ensuite on précise ces résultats lorsque $\gamma$ est une courbe intégrale et on prouve le théorème 1. La deuxième partie est essentiellement consacrée à la preuve du théorème 2 et de son corollaire.

Les trois auteurs remercient vivement la C.E.E. pour le support financier qu'elle leur a apporté pendant la préparation de ce travail, par l'intermédiaire du réseau T.M.R..

\section{Tangentes itérées, oscillation, spiralement}

Dans tout ce chapitre $M$ désigne une variété analytique (non singulière) de dimension $m$ et $\gamma$ désigne une application analytique non constante de $\mathbb{R}^{+}$dans $M$ qui possède un unique point $\omega$-limite $p=\lim _{t \longrightarrow \infty} \gamma(t)$. Pour décrire le comportement analytique de $|\gamma|$, l'image de $\gamma$, au voisinage de $p$ nous utilisons quatre concepts: tangentes itérées, contact plat, oscillation et spiralement. Dans la partie 1 de ce chapitre, nous étudions les relations qui les lient dans le cas général et dans la partie 2 nous précisons ces résultats lorsque $\gamma$ est une courbe intégrale d'un champ de vecteurs analytique réel.

\section{Courbes paramétrées}

Les définitions que nous allons donner dans ce paragraphe reposent sur les éclatements ponctuels et sur quelques propriétés classiques des ensembles semi-analytiques que l'on peut trouver dans $\left[\mathrm{Hi}_{1}\right],\left[\mathrm{Lo}_{2}\right]$.

1.1. Eclatement ponctuel et tangentes itérées. Précisons tout d'abord quelques notations et définitions. Une carte affine centrée en un point $p$ de $M$ est un difféomorphisme analytique $x=\left(x_{1}, x_{2}, \cdots, x_{m}\right)$ d'un voisinage $U$ de $p$ sur $\mathbb{R}^{m}$ tel que $x(p)=0$ (on dit "affine" pour rappeller que l'image de la carte est $\mathbb{R}^{m}$, quoique les changements de cartes ne seront pas linéaires). Un diviseur à croisements normaux $E$ de $M$ est une union finie d'hypersurfaces analytiques lisses vérifiant: en tout point $p$ de $M$, il existe une carte affine centrée en $p$ et un entier $\nu \geq 0$ telle que $E \cap U=\left\{x_{1} x_{2} \cdots x_{\nu}=0\right\}$. Une orientation de $M$ en $p$ adaptée à $E$ est le (choix du) germe $M^{+}$en $p$ d'une composante connexe de $U \backslash E$. Rappelons que l'éclatement de centre $p$ de $M$ est un morphisme analytique, surjectif, propre $\pi: M_{1} \longrightarrow M$ dont la restriction à $M \backslash \pi^{-1}\{p\}$ est un isomorphisme sur $M \backslash\{p\}$ possédant la propriété suivante. En tout point $p_{1}$ de $\pi^{-1}(p)$ il existe une carte 
affine $\left((x, z), U_{1}\right), x=\left(x_{1}, x_{2}, \cdots, x_{m-1}\right)$ centrée en $p_{1}$ et une carte affine centrée en $p$ telles que

$$
\pi(x, z)=(z x, z), \quad U_{1} \cap \pi^{-1}(p)=\{z=0\} .
$$

Notons que la restriction de $\pi$ à $U_{1} \equiv \mathbb{R}^{m}$ est un morphisme algébrique sur $U \equiv$ $\mathbb{R}^{m}$ et que le diviseur exceptionnel $\pi^{-1}(p)$ peut être identifié à l'espace projectif $\mathbb{R} P(m-1)$. Si $E$ est un diviseur à croisements normaux de $M, E_{1}=\pi^{-1}(E \cup\{p\})$ est un diviseur à croisements normaux de $M_{1}$. Une orientation $M_{1}^{+}$de $M_{1}$ en un point $p_{1}$ de $E_{1}$ adaptée à $E_{1}$ est dite compatible avec une orientation $M^{+}$de $M$ en $p$ adaptée à $E$ si $\pi\left(M_{1}^{+}\right) \subseteq M^{+}$.

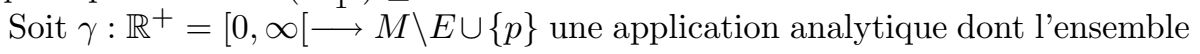
$\omega$-limite est le point $p$, c'est-à-dire:

$$
p=\omega(\gamma)=\lim _{t \longrightarrow \infty} \gamma(t)=\bigcap_{t \geq 0} \overline{\gamma((t, \infty))}
$$

Soit $\gamma_{1}=\pi^{-1} \circ \gamma$ le relevé de $\gamma$ par $\pi$ l'éclatement de centre $p$, i.e $\pi\left(\gamma_{1}(t)\right)=\gamma(t)$ pour $t \geq 0$. Si $\gamma_{1}$ possède un seul point $\omega$-limite $p_{1}=\omega\left(\gamma_{1}\right)$ on dit (naturellement) que $\gamma$ possède une tangente en $p$ et que $p_{1} \in \mathbb{R} P(m-1)$ est la (direction de la) tangente à $\gamma$ en $p$. Posons $M_{0}=M, p_{0}=p, E_{0}=\emptyset, \pi_{1}=\pi$.

Définition. On dit que $\gamma$ a (la propriété) des tangentes itérées s'il existe une suite:

$$
M_{0} \stackrel{\pi_{1}}{\longleftarrow} M_{1} \stackrel{\pi_{2}}{\longleftarrow} M_{2} \longleftarrow \cdots M_{n-1} \stackrel{\pi_{n}}{\longleftarrow} M_{n} \longleftarrow \cdots
$$

d'éclatements $\pi_{n}$ de centres $p_{n-1}$ telle que le relevé $\gamma_{n}=\pi_{n}^{-1} \circ \gamma_{n-1}$ possède un unique point $\omega$-limite $p_{n}=\omega\left(\gamma_{n}\right)$. La suite $T I(\gamma)=\left\{p_{n}\right\}$ est la suite des points infiniment proches de $\gamma=\gamma_{0}$. Notons, pour $n \geq 1, E_{n}=\pi_{n}^{-1}\left(E_{n-1} \cup\left\{p_{n-1}\right\}\right)$ et $M_{n}^{+}$l'orientation de $M_{n}$ adaptée à $E_{n}$ telle que $M_{n}^{+}$contient le germe de $\left|\gamma_{n}\right|$ en $p_{n}$. La suite $T I^{+}(\gamma)=\left\{\left(p_{n}, M_{n}^{+}\right)\right\}$est la suite orientée des points infiniment proches de $\gamma$. (Notons que lorsque $p \notin|\gamma|=$ image de $\gamma$ et que $\omega(\gamma)=\{p\}$, alors le germe en $p$ de $|\gamma|$ coïncide avec "l'image du germe" en $t=\infty$ de $\gamma$ ).

1.2. Oscillation. Dans tout ce paragraphe $\gamma$ désigne encore une application analytique de $\mathbb{R}^{+}$dans $M \backslash\{p\}$ telle que $\omega(\gamma)=p$.

Définition. Soit $H$ une hypersurface semi-analytique de $M$. On dit que $\gamma$ est oscillante en p par rapport à $H$ si $|\gamma|$ n'est pas contenu dans $H$ et si $|\gamma| \cap H$ est un ensemble infini. S'il n'existe pas une telle hypersurface nous dirons que $|\gamma|$ est non oscillante en $p$.

Ce concept d'oscillation d'une courbe est étudié quantitativement dans le cadre global par D. Novikov et S. Yakovenko dans [No-Ya].

Proposition. Si $|\gamma|$ est non oscillante en p, elle possède la propriété des tangentes itérées en $p$. 

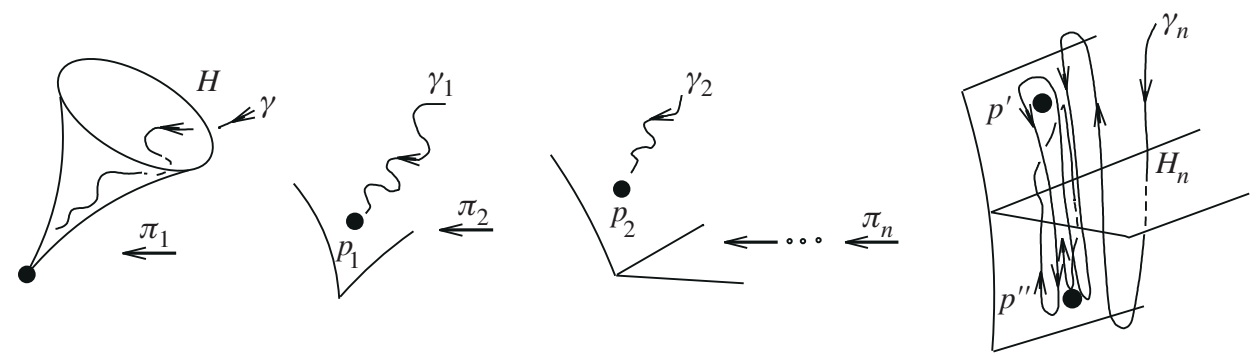

Figure 0.

La proposition réciproque n'est pas vraie en général. Par exemple la courbe $t \mapsto(1 / t, \exp (-t) \sin t)$ possède des tangentes itérées en $(0,0)$ et est oscillante par rapport à la droite $y=0$. Dans la deuxième partie de ce chapitre nous étudierons cette réciproque lorsque $\gamma$ est une courbe intégrale d'un champ de vecteurs analytique.

Preuve. Nous allons montrer la proposition contraposée. Posons $M_{0}=M, p_{0}=p$, $\gamma_{0}=\gamma$ et supposons qu'il existe une suite d'éclatements $\pi_{k}: M_{k} \rightarrow M_{k-1}$ pour $k=1,2, \ldots, n$ de centres $p_{k-1}$, telle que, pour $k=1,2, \ldots, n-1$, le relevé $\gamma_{k}=$ $\pi_{k}^{-1} \circ \gamma_{k-1}$ de $\gamma_{k-1}$ possède un unique point $\omega$-limite $p_{k}=\omega\left(\gamma_{k}\right)$ et telle que $\gamma_{n}=\pi_{n}^{-1} \circ \gamma_{n-1}$ possède au moins deux points $\omega$-limites distincts $p_{n}^{\prime}, p_{n}^{\prime \prime}$. Puisqu'il s'agit de prouver un résultat local nous pouvons supposer que $M_{0}=\mathbb{R}^{m}$ et que les deux points $p_{n}^{\prime}, p_{n}^{\prime \prime}$ sont contenus dans une même carte affine $x: U \longrightarrow \mathbb{R}^{m}$ telle que $\pi_{1} \circ \pi_{2} \circ \cdots \circ \pi_{n} \circ x^{-1}$ soit une application polynomiale. D'après le théorème de Tarski-Seidenberg [Ta] sur les projections d'ensembles semi-algébriques, il suffit de construire une hypersurface algébrique $H_{n}$ dans $U \equiv \mathbb{R}^{m}$ non contenue dans le diviseur telle que \# $H_{n} \cap\left|\gamma_{n}\right|=\infty$ et $\left|\gamma_{n}\right| \not \subset H_{n}$. En effet, sa projection par $\pi_{1} \circ \pi_{2} \circ \cdots \circ \pi_{n} \circ x^{-1}$ sera une hypersurface semi-algébrique $H$ telle que $\# H \cap \gamma=\infty$ et $|\gamma| \not \subset H$. Soient $\left(x_{1}, x_{2}, \cdots, x_{m-1}, z\right)$ des coordonnées affines sur $U$ telles que:

$$
\{z=0\}=\pi_{n}^{-1}\left(p_{n-1}\right) \quad \text { et } \quad p_{n}^{\prime}, p_{n}^{\prime \prime} \in B=\left\{x_{1}^{2}+x_{2}^{2}+\cdots+x_{m-1}^{2}+z^{2}<1\right\} .
$$

Si $\left|\gamma_{n}\right|$ coupe une infinité de fois la sphère $\partial B$ on prend $H_{n}=\partial B$. Si ce n'est pas le cas, soit $H_{n}$ un hyperplan $f\left(x_{1}, \cdots, x_{m-1}, z\right)=\alpha_{1} x_{1}+\cdots+\alpha_{m-1} x_{m-1}+\beta=0$ tel que $f\left(p_{n}^{\prime}\right) . f\left(p_{n}^{\prime \prime}\right)<0$. Les points $p_{n}^{\prime}, p_{n}^{\prime \prime}$ appartenant à deux composantes connexes distinctes de $B \backslash H_{n},|\gamma|$ coupe une infinité de fois leur "frontière commune" $B \cap H_{n}$.

Remarque. Le concept "osciller" est stable par éclatement. Si $\gamma$ est oscillante par rapport à $H$, alors $\pi^{-1} \circ \gamma=\gamma_{1}$ est oscillante par rapport à $\pi^{-1}(H)=$ $H_{1}$. Par contre, il ne l'est pas, à priori, par effondrement puisque l'image directe 
par éclatement ponctuel d'une hypersurface analytique n'est pas en général semianalytique $\left[\mathrm{Lo}_{2}\right],\left[\mathrm{Hi}_{1}\right]$. Dans la preuve précédente, on contourne cette difficulté en travaillant dans des cartes affines pour se placer dans le cadre algébrique. Nous aurions pu faire une théorie semblable dans le cadre sous-analytique. Les deux théories ne sont pas équivalentes. Il nous semble qu'elles coïncident lorsqu'il s'agît de trajectoires de champs de vecteurs (mais nous ne l'avons pas démontré).

1.3. Contact plat avec une demi-branche analytique. Soit $c$ une application analytique non constante de $\mathbb{R}$ dans $M$ et soit $E$ un diviseur à croisements normaux. Le germe $\Gamma$ de $c(] 0, \varepsilon[), \varepsilon>0$, en $p=c(0)$ est appelé une demi-branche analytique en $p$. Elle est dite adaptée à $E$ si $\Gamma \cap E=\emptyset$. On dit que $\Gamma$ est lisse relativement à $E$ s'il existe une carte affine $\left(\left(x_{1}, \ldots, x_{m-1}, z\right), U\right)$ centrée en $p$ telle que

$$
\Gamma=\left\{x_{1}=x_{2}=\cdots=x_{m-1}=0, z>0\right\} \text { et } E \cap U=\{z=0\} .
$$

Soit $\pi_{1}: M_{1} \longrightarrow M=M_{0}$ l'éclatement de centre $p=p_{0}$. On sait que $c$ possède un unique relèvement $c_{1}$ par $\pi, c(s)=\pi \circ c_{1}(s)$. La contre-image $\Gamma_{1}=\pi_{1}^{-1}\left(\Gamma_{0}\right)$, avec $\Gamma_{0}=\Gamma$, est la demi-branche analytique en $p_{1}=c_{1}(0)$ adaptée à $E_{1}=\pi^{-1}(E \cup\{p\})$ qui est le germe de $c_{1}(] 0, \varepsilon[)$ en $p_{1}$. En itérant cette construction, on obtient une suite d'éclatements:

$$
M_{0} \stackrel{\pi_{1}}{\longleftarrow} M_{1} \stackrel{\pi_{2}}{\longleftarrow} M_{2} \cdots \longleftarrow M_{n-1} \stackrel{\pi_{n}}{\longleftarrow} M_{n} \cdots
$$

de centre des points $p_{n-1}$ et une suite de demi-branches analytiques $\Gamma_{n}, n \geq 0$, aux points $p_{n}$ adaptées aux diviseurs $E_{n}=\pi_{n}^{-1}\left(E_{n-1}\right)$. La suite $\left\{p_{n}\right\}, n \geq 1$ est la suite des points infiniment proches au sens de 1.1 de la courbe $\sigma: t \mapsto c(1 / t)$, $t>0$ et nous écrirons:

$$
T I(\Gamma)=T I(\sigma)=\left\{p_{n}\right\} \quad \text { et } \quad T I^{+}(\Gamma)=T I^{+}(\sigma)=\left\{\left(p_{n}, M_{n}^{+}\right)\right\} .
$$

Rappelons ([Lo2], $\left[\mathrm{Hi}_{1}\right]$ ) les propriétés suivantes des demi-branches analytiques qui sont bien connues:

i) Il existe $n_{0}$ tel que, pour $n \geq n_{0}, \Gamma_{n}$ soit lisse relativement à $E_{n}$.

ii) Si $\Gamma^{\prime}$ est une demi-branche analytique telle que $T I^{+}\left(\Gamma^{\prime}\right)=T I^{+}(\Gamma)$ alors $\Gamma^{\prime} \equiv$ $\Gamma$.

iii) Soit $A$ un sous-ensemble semi-analytique de $M$. Alors $\Gamma$ est contenu dans $\bar{A}$ si et seulement si $p_{n}$ appartient à l'adhérence de $\left(\pi_{1} \circ \pi_{2} \circ \cdots \circ \pi_{n}\right)^{-1}(A) \cap M_{n}^{+}$ pour tout $n \geq 0$.

Dans toute la suite de ce paragraphe, $\gamma$ désigne une application analytique de $\mathbb{R}^{+}$ dans $M \backslash\{p\}$ telle que $\omega(\gamma)=p$. 
Définition. Soit $\Gamma$ une demi-branche analytique en $p$. On dit que $\gamma$ a un contact plat avec $\Gamma$ si $\gamma$ a la propriété des tangentes itérées et si $\mathrm{TI}^{+}(\gamma)=T I^{+}(\Gamma)$.

Par définition, ce concept est stable par éclatement et par effondrement.

Proposition (propriété caractéristique). Soit $\Gamma$ une demi-branche analytique lisse en $p$ adaptée à $E$, i.e. $\Gamma=\left\{x_{1}=\cdots=x_{m-1}=0, z>0\right\}, E \cap U=\{z=0\}$ dans une carte affine $U$ centrée en $p$. Alors $\gamma$ a un contact plat avec $\Gamma$ si et seulement si on a:

$$
\lim _{t \rightarrow \infty} z(t)=0 \quad, \quad z(t)>0 \quad \text { et } \quad \lim _{t \rightarrow \infty} \frac{\|x(t)\|}{z(t)^{n}}=0 \quad \text { pour } \quad n \geq 0,
$$

en écrivant $\gamma(t)=(x(t), z(t)),\|x\|^{2}=x_{1}^{2}+x_{2}^{2}+\cdots+x_{m-1}^{2}$.

L'existence des tangentes itérées n'implique pas le contact plat comme le montre l'exemple $\gamma: t \rightarrow\left(t^{-1}, t^{-\lambda}\right), \lambda \in \mathbb{R}^{+} \backslash \mathbb{Q}$. En effet $T I(\gamma)$ contient un nombre infini de "coins" du diviseur déterminés par le développement en fraction continue de $\lambda$.

Preuve. Soit $T I(\Gamma)=\left\{p_{n}\right\}$ la suite de points infiniment proches de $\Gamma$ et soit $\left\{\pi_{n}\right\}$ la suite d'éclatements de centre les points $p_{n-1}$. Il existe une carte affine en $p_{n}$, $\left((u, z), U_{n}\right), u=\left(u_{1}, u_{2}, \cdots, u_{m-1}\right)$ telle que:

$$
\pi_{1} \circ \pi_{2} \circ \cdots \circ \pi_{n}(u, z)=\left(z^{n} u, z\right) .
$$

Soit $\gamma_{n}=\pi_{n}^{-1} \circ \gamma_{n-1}$ le relevé de $\gamma=\gamma_{0}$ par cette composition d'éclatements. En écrivant $\gamma_{n}(t)=(u(t), z(t))$ la proposition est une conséquence immédiate des définitions et de l'égalité $x(t)=z(t)^{n} u(t)$.

1.4. Spiralement. Le concept de "spiraler autour de..." en dimension 2 et 3 fait partie du langage courant, nous allons le préciser. Dans tout ce paragraphe $\gamma$ est, comme dans les paragraphes précédents, une application analytique de $\mathbb{R}^{+}$ dans $M \backslash\{p\}$ telle que $\omega(\gamma)=p$. Nous dirons qu'une hypersurface semi-analytique $T$ est transversalement orientée par $\gamma$ si $T$ est connexe, lisse, orientable et si $\gamma$ coupe transversalement $T$ une infinité de fois toujours dans le même sens pour $t$ assez grand. C'est-à-dire que $T$ possède un voisinage ouvert connexe $U$ dans $M$ tel que $U \backslash T$ a deux composantes connexes $U^{+}, U^{-}$et tel que pour $t$ assez grand et $\gamma(t) \in T$ on a $\gamma\left(t+\varepsilon_{t}\right) \in U^{+}$et $\gamma\left(t-\varepsilon_{t}\right) \in U^{-}$pour $\varepsilon_{t}>0$ assez petit.

Spiralement en dimension 2. Si $m=\operatorname{dim} M=2$ on dit que $\gamma$ spirale autour de $\{p\}$ si toute demi-branche analytique $T$ en $p$ est transversalement orientée par $\gamma$ (figure 1).

Soit $\left(x_{1}, x_{2}\right)$ une carte affine centrée en $p$ et soient $(r, \theta)$ les coordonnées polaires correspondantes. Si $\gamma$ spirale autour de $p$, l'angle $\theta(t)=\oint_{\gamma \mid[0, t]} d \theta$ tend vers plus 


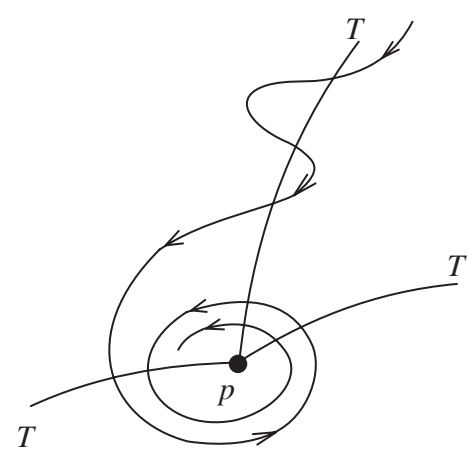

Spiralement

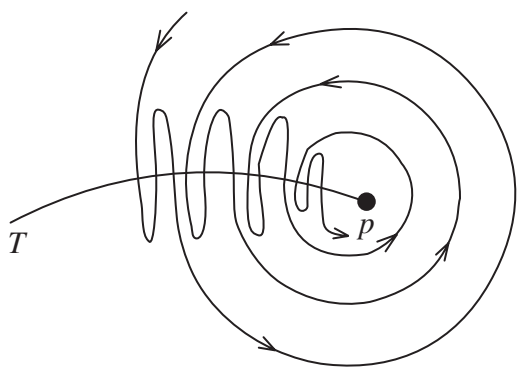

Nonspiralement

Figure 1.

ou moins l'infini si $t$ tend vers l'infini. Cette dernière propriété n'implique pas en général que $\gamma$ spirale autour de $p$. Cependant, nous verrons dans I.2 que c'est le cas lorsque $\gamma$ est une courbe intégrale d'un champ de vecteurs analytique.

Spiralement en dimension 3. En dimension 2, la définition de spiralement repose sur la notion de demi-branche analytique transversalement orientée par $\gamma$. En dimension 3 elle va reposer sur la notion de triangle analytique transversalement orienté par $\gamma$ de côté une demi-branche analytique. Soit $\Gamma$ une demi-branche analytique en un point $p$ et soit $c:] 0, \varepsilon\left[\longrightarrow M^{3}\right.$ un représentant de $\Gamma$ où $c$ est une application analytique de $\mathbb{R}$ dans $M^{3}$. Un triangle semi-analytique de côté $\Gamma$ est un couple $(T, W)$ où:

i) $W$ est un voisinage tubulaire ouvert semi-analytique de $c(] 0, \varepsilon[)$. C'est-à-dire que $W$ est un ouvert semi-analytique image de $] 0, \varepsilon\left[\times \mathbb{R}^{2}\right.$ par un plongement analytique $j$ tel que $j(t, 0)=c(t)$.

ii) $T$ est une surface semi-analytique lisse, connexe contenue dans $W \backslash c(] 0, \varepsilon[)$ telle que le triplet $(W, T, c(] 0, \varepsilon[))$ soit homéomorphe à (] $0, \varepsilon\left[\times \mathbb{R}^{2},\right] 0, \varepsilon\left[\times \mathbb{R}^{+} \times\{0\}\right.$, ] $0, \varepsilon[\times\{0\} \times\{0\})$.

Deux triangles semi-analytiques $\left(T_{1}, W_{1}\right),\left(T_{2}, W_{2}\right)$ sont dits compatibles si $W_{1}=$ $W_{2}=W$ et soit $T_{1}=T_{2}$ soit il existe un homéomorphisme de $\left(W, T_{1}, T_{2}, c([0, \varepsilon])\right)$ $\operatorname{sur}(] 0, \varepsilon\left[\times \mathbb{R}^{2},\right] 0, \varepsilon\left[\times \mathbb{R}^{+},\right] 0, \varepsilon\left[\times \mathbb{R}^{-},\right] 0, \varepsilon[\times\{0\} \times\{0\})$.

Compte tenu de la structure conique $\left[\mathrm{Lo}_{2}\right]$ des ensembles semi-analytiques, les propriétés i), ii) de $(T, W)$ se germifient en $p$ et dans leurs énoncés nous pouvons remplacer $c(] 0, \varepsilon[)$ par $\Gamma$. Ceci justifie l'expression triangle analytique de côté $\Gamma$. 


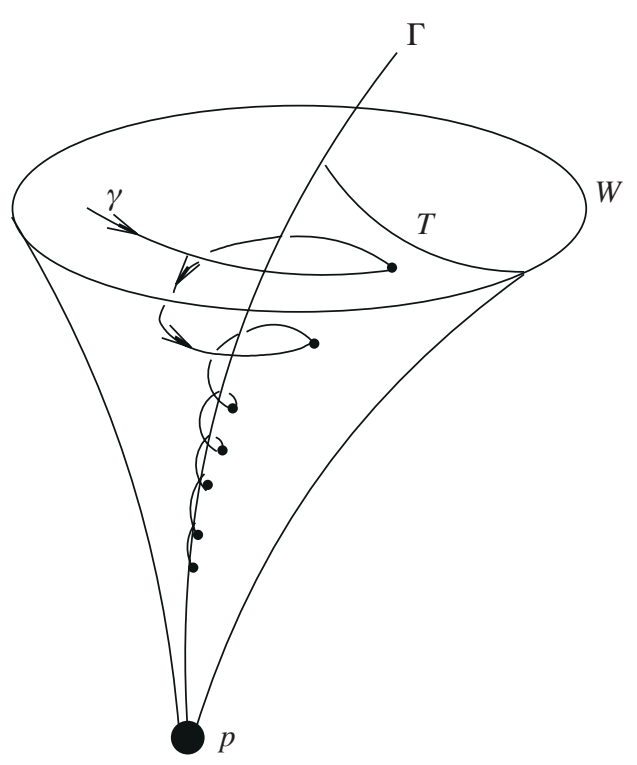

Figure 2.

Définition. Supposons $\operatorname{dim} M=3$ et soit $\Gamma$ une demi-branche analytique en $p$. On dit que $\gamma$ spirale autour de $\Gamma$ ( $\Gamma$ est un axe de spiralement pour $\gamma$ ) si pour tout triangle analytique $(T, W)$ de côté $\Gamma$, on a les propriétés: $T$ est transversalement orienté par $\gamma$ et $\gamma(t)$ appartient à $W$ pour $t$ assez grand (figure 2).

Supposons que $\Gamma$ soit un axe de spiralement lisse de $\gamma$. Soit $((x, z), U)$ une carte affine centrée en $p$ telle que $\Gamma=\left\{x_{1}=x_{2}=0, z>0\right\}$. Notons $\gamma(t)=\left(x_{1}(t)\right.$, $\left.x_{2}(t), z(t)\right)$ et soit $\gamma_{0}: t \mapsto\left(x_{1}(t), x_{2}(t)\right)$ la projection de $\gamma$ sur $z=0$. A toute demi-branche analytique $T_{0} \subset\{z=0\}$ au point $p$ correspond le triangle analytique $(W, T)$ défini par

$W=\left\{\left(x_{1}, x_{2}, z\right): x_{1}^{2}+x_{2}^{2}<1, z>0\right\}$ et $T=\left\{\left(x_{1}, x_{2}, z\right) \in W:\left(x_{1}, x_{2}\right) \in T_{0}\right\}$.

Ainsi, $T_{0}$ est transversalement orienté par $\gamma_{0}$ et $\omega\left(\gamma_{0}\right)=p$, la projection $\gamma_{0}$ de $\gamma$ spirale autour de $p$. La réciproque n'est pas vraie en général, par exemple lorsque $|\gamma|$ est contenu dans le cone $x^{2}+y^{2}=z^{2}$.

Dans le cas général (axe de spiralement $\Gamma$ non nécessairement lisse) la définition du spiralement axial n'est pertinente que s'il existe "beaucoup" de triangles analytiques de côté une demi-branche analytique $\Gamma$ fixée. Le lemme suivant montre que c'est le cas.

Lemme. Soit $\Gamma$ une demi-branche analytique en $p=p_{0} \in M^{3}$ et soit:

$$
M_{0} \stackrel{\pi_{1}}{\longleftarrow} M_{1} \stackrel{\pi_{2}}{\longleftarrow} M_{2} \longleftarrow \cdots
$$




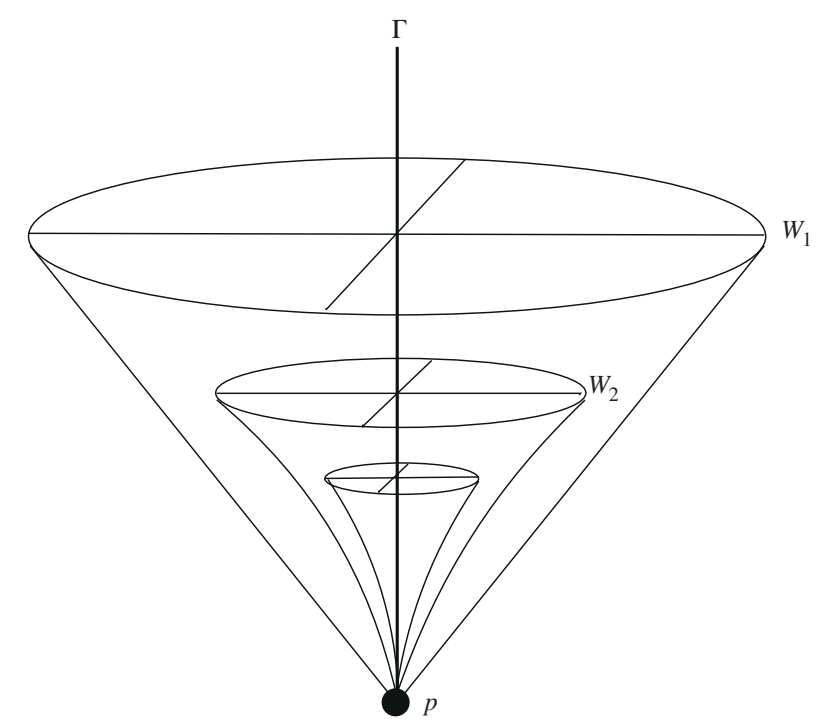

Figure 3.

la suite d'éclatements de centre $p_{0}$ et les points $p_{1}, p_{2}, \ldots$ de $T I(\Gamma)$. Il existe une suite d'ouverts semi-analytiques $\left\{W_{n}\right\}$ de $M_{0}$ qui possède les propriétés suivantes: i) Pour tout $n$ le germe de $W_{n}$ en $p$ contient $\Gamma$, $p$ n'appartient pas à $W_{n}$ et $W_{n+1} \subset$ $W_{n}$.

ii) Si $A$ est un ensemble semi-analytique tel que $\bar{A} \cap \Gamma=\emptyset$, alors $A \cap W_{n}=\emptyset$ pour $n$ assez grand.

iii) Soit $A$ un ensemble semi-analytique de dimension 2 tel que $A \cap \Gamma=\emptyset$ et $\Gamma \subset \bar{A}$. Il existe $n_{0}$ tel que, $S_{1}, S_{2}, \cdots, S_{r}$ désignant les composantes connexes de $A \cap W_{n_{0}}$, les $\left(S_{i} \cap W_{n}, W_{n}\right)$ sont des triangles semi-analytiques de côté $\Gamma$ deux à deux compatibles pour $i=1,2, \ldots, r$ et $n \geq n_{0}$.

iv) Soient $W_{n, k}$ et $\Gamma_{k}$ les images réciproques de $W_{n}$ et $\Gamma$ par $\pi_{1} \circ \pi_{2} \circ \ldots \circ \pi_{k}$. Etant donné $n>0$, il existe $k_{0}, n^{\prime}>n$ et une carte affine $\left(U,\left(x_{1}, x_{2}, z\right)\right)$ en $p_{k_{0}}$ tels que:

$W_{n, k_{0}} \cap U=\{z>0\} \quad, \quad \Gamma_{k_{0}}=\left\{x_{1}=x_{2}=0, z>0\right\}, \bar{W}_{n^{\prime}, k_{0}} \cap\{z=0\}=\left\{p_{k_{0}}\right\}$.

Ce lemme est une conséquence de l'existence de triangulations semi-analytiques des ensembles semi-analytiques $\left[\mathrm{Lo}_{2}\right],\left[\mathrm{Hi}_{1}\right]$. Il peut être aussi prouvé de façon plus élémentaire. Les assertions i), ii), iv) sont évidentes. L'assertion iii) se montre en utilisant les équations de $A$. En fait, lorsque $\Gamma$ est désingularisée par un morphisme $\pi$ nous pouvons essentiellement supposer que les $W_{n}$ sont les projections par $\pi$ des $W_{n}^{\prime}=\left\{x^{2}+y^{2}<z^{2 n} ; 0<z<1 / n\right\}$.

D'après iv), si $\gamma: \mathbb{R}^{+} \longrightarrow \mathbb{R}^{3}, \gamma(0)=p$ est une courbe analytique ayant un contact plat avec $\Gamma$, alors, quelque soit $n$, il existe $t_{n}$ tel que $\gamma(t)$ appartient à $W_{n}$ pour $t>t_{n}$. La proposition suivante précise le concept de spiralement axial. 
Proposition. Si $\gamma$ spirale autour d'une demi-branche analytique $\Gamma$ en $p$, alors $\gamma$ a un contact plat avec $\Gamma$.

Preuve. Raisonnons par l'absurde en reprennant les notations du lemme ci-dessus. Si $\gamma$ n'a pas un contact plat avec $\Gamma$, il existe $k$ tel que l'ensemble $\omega$-limite, $\omega\left(\gamma_{k}\right)$ de $\gamma_{k}=\left(\pi_{1} \circ \pi_{2} \circ \cdots \circ \pi_{k}\right)^{-1}(\gamma)$ contienne un point $q \neq p_{k}$. D'après l'assertion iv) du lemme précédent il existe $n$ et une suite $\left\{t_{m}\right\}$ tendant vers l'infini telle que $\gamma_{k}\left(t_{m}\right)$ n'appartient pas à $W_{n}$. Ainsi $\gamma(t)$ ne peut pas appartenir à $W_{n}$ pour $t$ assez grand.

Remarque. La définition que nous avons donnée du spiralement axial est très restrictive. Il est possible de l'affaiblir, par exemple en supprimant la condition " $\gamma(t) \in W$ pour $t$ assez grand", ou en remplaçant "pour tout $(T, W) \cdots$ " par "il existe un $(T, W) \cdots "$. Nous verrons, dans la suite, que toutes ces définitions sont équivalentes lorsque $\gamma$ est une courbe intégrale d'un champ de vecteurs analytique en dimension 3 .

\section{Courbes intégrales}

Dans toute cette partie $X$ désigne un champ de vecteurs analytique sur $M^{m}$ dont $p$ est un point singulier et $\gamma$ désigne une demi-courbe intégrale, $\gamma: t \mapsto \gamma(t)$, $t \geq 0$ telle que $p=\omega(\gamma)$. Nous allons essentiellement étudier comment sont alors reliés les concepts: tangentes itérées, oscillation, spiralement en dimension 2 et 3. Auparavant, nous allons montrer des propriétés du contact plat qui sont vraies en toute dimension.

2.1. Contact plat entre une courbe intégrale et une demi-branche analytique. Les résultats énoncés dans les deux propositions suivantes sont peut-être déjà (bien) connus. Par exemple, celui de la première proposition peut certainement se déduire d'un théorème classique de Borel [Bo], celui de la deuxième parait aussi classique, mais nous ne l'avons pas trouvé dans la littérature usuelle. Les démonstrations que nous en proposons sont géométriques. Elles utilisent essentiellement la "désingularisation" des demi-branches analytiques. La proposition 2.1.1. est un outil essentiel dans la preuve du théorème 1 et la proposition 2.1.2. dans la preuve du théorème 2 .

2.1.1. Proposition. Soit $\gamma$ une demi-courbe intégrale d'un champ de vecteurs analytique $X$. Si $\gamma$ a un contact plat en $p$ avec une demi-branche analytique $\Gamma$, alors $\Gamma$ est une courbe invariante par $X$.

Avant d'aborder la preuve de cette proposition, précisons quelques notations qui seront utiles dans toute la suite. Soit $M=M_{0} \stackrel{\pi_{1}}{\longleftarrow} M_{1} \stackrel{\pi_{2}}{\longleftarrow} M_{2} \longleftarrow \cdots$ la suite d'éclatements de centre $p_{0}=p, p_{1}, \ldots$ où $T I(\Gamma)=\left\{p_{n}\right\}$. Pour $k \geq 1$ il existe un 
unique champ de vecteurs analytique $X_{k}$ sur $M_{k}$ relié à $X_{k-1}$ par $\pi_{k}$, avec $X_{0}=X$. On dit que $X_{k}$ est le relevé de $X_{k-1}$ par $\pi_{k}$. Le diviseur $E_{k}=\left(\pi_{1} \circ \pi_{2} \cdots \circ \pi_{k}\right)^{-1}\left(p_{0}\right)$ est invariant par $X_{k}$. Une application $\gamma_{k-1}: \mathbb{R}^{+} \longrightarrow M_{k-1} \backslash E_{k-1}, t \mapsto \gamma_{k-1}(t)$, est une courbe intégrale de $X_{k-1}$ si et seulement si son relevé $\gamma_{k}=\pi_{k}^{-1} \circ \gamma_{k-1}$ est une courbe intégrale de $X_{k}$.

Preuve. Supposons $T I^{+}(\Gamma)=T I^{+}(\gamma)$. Puisque les propriétés tangentes itérées, contact plat sont invariantes par éclatements, effondrements et que $\Gamma$ est l'image d'une demi-branche orientée lisse par le composé d'un nombre fini d'effondrements, on peut supposer que $\Gamma=\left\{x_{1}=x_{2}=\cdots=x_{m-1}=0, z>0\right\}$ et que $E_{0}=\{z=0\}$ est invariant par le champ de vecteurs $X$. C'est-à-dire que

$$
X=\sum_{i=1}^{m-1}\left(a_{i}(z)+\sum_{j=1}^{m-1} x_{j} a_{i, j}(x, z)\right) \frac{\partial}{\partial x_{i}}+z a(x, z) \frac{\partial}{\partial z}
$$

les $a, a_{i}, a_{i, j}$ étant analytiques au voisinage de $0 \in \mathbb{R}^{m}$. Si $a_{i} \equiv 0$ pour $i=1$, $2, \cdots, m-1$, la demi-branche $\Gamma$ est invariante par $X$. Supposons que ce ne soit pas le cas. Il existe $n \geq 0$ tel que l'on ait pour chaque $i$ :

$$
a_{i}(z)=z^{n} b_{i}(z), \quad \Sigma b_{i}^{2}(0) \neq 0 \quad, \quad b_{i}(z) \in \mathbb{R}\{z\} .
$$

Il existe une carte $(y, z)$ centrée en $p_{n}$ telle que:

$$
(y, z)=\left(z^{n} x, z\right), \quad y=\left(y_{1}, y_{2}, \cdots, y_{m-1}\right) .
$$

Le relevé $X_{n}$ de $X=X_{0}$ s'écrit dans ces coordonnées:

$$
X_{n}=\sum_{i=1}^{m-1}\left(b_{i}(z)+\sum_{j=1}^{m-1} y_{j} b_{i, j}\left(z^{n} y, z\right)\right) \frac{\partial}{\partial y_{i}}+z a\left(z^{n} y, z\right) \frac{\partial}{\partial z}
$$

où les $b_{i, j}$ sont analytiques au voisinage de 0 . On a alors:

$$
X_{n}\left(p_{n}\right)=X_{n}(0)=\sum_{i=1}^{m-1} b_{i}(0) \frac{\partial}{\partial y_{i}} \neq 0
$$

ce qui est incompatible avec l'hypothèse $\omega\left(\gamma_{n}\right)=p_{n}$.

2.1.2. Proposition. Soient $X$ un champ de vecteurs analytique sur $M^{m}$ singulier en $p, \Gamma$ une demi-branche analytique en $p$ invariante par $X$ et soit $v(\Gamma)$ un vecteur tangent à $\Gamma$ en $p$. Alors, $v(\Gamma)$ est un vecteur propre de la partie linéaire $D X(p)$. De plus, la valeur propre $\mu(\Gamma)$ correspondant à $v(\Gamma)$ est nulle s'il existe une demicourbe intégrale positive de $X$ distincte de $\Gamma$ ayant un contact plat avec $\Gamma$ en $p$. 
Preuve. La première assertion de la proposition est une conséquence de la remarque suivante. Si $\pi_{1}: M_{1} \longrightarrow M$ est l'éclatement de centre $p$, alors un point $q \in E_{1}=$ $\pi_{1}^{-1}(p)=\mathbb{R} P(m-1)$ est un point fixe de $X_{1}$ le relevé de $X$ si et seulement si $q \in \mathbb{R} P(n-1)$ est une direction propre de $D X(p)$. En effet, $p_{1}$ est un point singulier de $X_{1}$ si $p_{1}$ est la direction de la tangente à $\Gamma$ en $p$. La preuve de la seconde partie de la proposition repose essentiellement sur l'étude de l'évolution du spectre de $D X(p)$ par éclatement. Elle est décrite par l'assertion suivante.

Assertion. Soit $\left\{\mu_{1}, \mu_{2}, \ldots, \mu_{n-1}, \mu(\Gamma)\right\}$ le spectre de $D X(p)$ où $\mu(\Gamma)$ est la valeur propre correspondant à un vecteur $v(\Gamma)$ tangent à $\Gamma$ en p, i.e $p_{1}=[v(\Gamma)] \in$ $\mathbb{R} P(m-1)$. Alors $E_{1}=\pi^{-1}(p)$ est invariant par $X_{1}$ et on a:

$$
\begin{aligned}
\operatorname{Spec}\left(D X_{1}\left(p_{1}\right)\right)=\left\{\mu_{1}-\mu(\Gamma), \ldots, \mu_{m-1}-\right. & \mu(\Gamma), \mu(\Gamma)\} \\
& =\operatorname{Spec}\left(\left.D X_{1}\left(p_{1}\right)\right|_{E_{1}}\right) \cup\{\mu(\Gamma)\} .
\end{aligned}
$$

De plus si $H$ est une hypersurface lisse invariante par $X$, si $v(\Gamma)$ est tangent à $H$ et si $\mu_{1}$ correspond $\grave{a}$ un vecteur propre transverse $\grave{a} H$, alors $X_{1}$ est tangent au transformé strict $H_{1}$ de $H$ par $\pi_{1}$ et on a:

$$
\operatorname{Spec}\left(D X_{1}\left(p_{1}\right)\right)=\left\{\mu_{1}-\mu(\Gamma)\right\} \cup \operatorname{Spec}\left(\left.D X_{1}\left(p_{1}\right)\right|_{H_{1}}\right) .
$$

Pour prouver cette assertion choisissons des coordonnées $(x, z), x=\left(x_{1}, x_{2}, \cdots\right.$, $x_{m-1}$ ) en $p$ telles que $v(\Gamma)$ soit tangent à l'axe $x=0$. On écrit

$$
X=\sum_{i}\left(<a_{i}, x>+f_{i}(x, z)\right) \frac{\partial}{\partial x_{i}}+(\mu(\Gamma) z+<b, x>+\cdots) \frac{\partial}{\partial z}
$$

où $\cdots$ désigne des termes d'ordre $\geq 2$ et $<$, $>$ désigne le produit scalaire canonique sur $\mathbb{R}^{m-1}$. Dans ces coordonnées on a:

$$
D X(p)=\left(\begin{array}{cc}
A & 0 \\
b & \mu(\Gamma)
\end{array}\right) \quad \text { avec }\left\{\begin{array}{l}
a_{i}=\left(a_{i, 1}, a_{i, 2}, \cdots, a_{i, m-1}\right) \\
b=\left(b_{1}, b_{2}, \cdots, b_{m-1}\right)
\end{array}\right.
$$

et $A=\left(a_{i, j}\right)$. Soient $\left(x^{\prime}, z\right)$ des coordonnées en $p_{1}$ telles que $x=z x^{\prime}$. Dans ces coordonnées on a

$$
D X_{1}\left(p_{1}\right)=\left(\begin{array}{cc}
A-\mu(\Gamma) I_{m-1} & c \\
0 & \mu(\Gamma)
\end{array}\right) \quad \text { avec } \quad c_{m, i}=\frac{1}{2} \frac{\partial^{2} f_{i}}{\partial z^{2}} \quad(0,0) .
$$

Ceci prouve la première partie de l'assertion. Pour prouver la deuxième partie choisissons des coordonnées $(x, z)$ tels que $H=\left\{x_{1}=0\right\}$. Alors $\left\{x_{1}=0\right\}$ étant 
invariant par $X$ on a $a_{1}=0$ et $f_{1}$ est divisible par $x_{1}$, en particulier $c_{m, 1}=0$. On en déduit:

$$
\operatorname{Spec}\left(D X_{1}\left(p_{1}\right)\right)=\left(\mu_{1}-\mu(\Gamma)\right) \cup \operatorname{Spec}\left(\left.D X_{1}\left(p_{1}\right)\right|_{\left\{x_{1}^{\prime}=0\right\}}\right),
$$

ce qui prouve l'assertion puisque $\left\{x_{1}^{\prime}=0\right\}$ est le transformé strict $H_{1}$ de $H$.

Fin de la preuve de la proposition. Cette preuve se fait par récurrence sur $m$ la dimension de $M$ et par l'absurde. C'est-à-dire que nous allons monter que $\mu(\Gamma) \neq 0$ est incompatible avec l'hypothèse $\gamma, \Gamma$ sont des demi-courbes intégrales de $X$ ayant un contact plat et $|\gamma| \neq \Gamma$. Lorsque $m=1$, cette contradiction est évidente puisque la condition $\omega(\gamma)=\omega(\Gamma)$ implique $\gamma \equiv \Gamma$.

Supposons $m>1$ et $\mu(\Gamma) \neq 0$, alors $\mu(\Gamma)<0$ puisque $p=\omega(\gamma)$. Les courbes $|\gamma|, \Gamma$ sont tangentes à $W^{s}(X, p)$, la variété stable de $X$ en $p$. Ainsi $|\gamma|$ et $\Gamma$ sont contenues dans $W^{s}(X, p)$ qui est analytique lisse ([Po], [Ha]). Si $W^{s}(X, p)$ n'est pas de dimension $m$ on aboutit à une contradiction d'après l'hypothèse de récurrence. Ainsi on peut supposer que toutes les valeurs propres de $D X(p)$ ont une partie réelle négative.

Notons $\pi_{k}: M_{k} \longrightarrow M_{k-1}$ la suite d'éclatements de centres les points $p_{k} \in$ $T I(\Gamma)$ et posons $\Gamma=\Gamma_{0}, \gamma=\gamma_{0}, \Gamma_{k}=\pi_{k}^{-1}\left(\Gamma_{k-1}\right), \gamma_{k}=\pi^{-1}(\gamma)$, Puisque $\Gamma_{k}$ est invariante par $X_{k}$, le relevé de $X_{k-1}$, sa tangente $\tau\left(\Gamma_{k}\right)$ en $p_{k}$ est une direction propre de $D X_{k}\left(p_{k}\right)$ de valeur propre $\mu\left(\Gamma_{k}\right)$. En fait $p_{k+1}$ est le point de $\pi_{k}^{-1}\left(p_{k}\right) \equiv$ $\mathbb{R} P(m-1)$ correspondant à $\tau\left(\Gamma_{k}\right)$ et de plus $\Gamma_{k}, \gamma_{k}$ ont encore un contact plat. Distinguons deux cas:

$1^{e r}$ cas, $\Gamma$ est lisse. En choisissant des coordonnées $\left(x^{(k)}, z\right)$ en $p_{k}$ telles que $\Gamma_{k}=\left\{x^{(k)}=0\right\}$, on a, d'après l'assertion précédente (et avec les mêmes notations)

$$
\operatorname{Spec}\left(D X_{k}\left(p_{k}\right)\right)=\left\{\mu_{1}-k \mu(\Gamma), \cdots, \mu_{m-1}-k \mu(\Gamma)\right\} \cup\{\mu(\Gamma)\} .
$$

Ainsi, $\mu(\Gamma)$ étant strictement négatif, pour $k$ assez grand les $\mu_{j}-k \mu(\Gamma)$ ont tous des parties réelles positives. La variété stable $W^{s}\left(X_{k}, p_{k}\right)$ est de dimension 1 . On termine par récurrence.

$2^{\text {ème }}$ cas, $\Gamma$ singulière. Notons tout d'abord que $\mu\left(\Gamma_{k}\right)$ ne peut pas rester strictement négatif pour tout $k$. En effet, pour $k$ assez grand, $\Gamma_{k}$ est lisse et si $\mu\left(\Gamma_{k}\right)<0$ pour tout $k$ on retombe dans le cas précédent. Ainsi, il existe $\ell$ tel que $\mu\left(\Gamma_{\ell-1}\right)<0$ et $\mu\left(\Gamma_{\ell}\right)=0$. De plus, d'après le premier cas, on peut choisir $\ell$ tel que $\mu\left(\Gamma_{j}\right)=0$ pour $j \geq \ell$. D'après l'assertion on a:

$$
\operatorname{Spec}\left(D X_{\ell}\left(p_{\ell}\right)\right)=\left\{\mu\left(\Gamma_{\ell-1}\right)\right\} \cup \operatorname{Spec}\left(\left.D X_{\ell}\left(p_{\ell}\right)\right|_{E_{\ell}}\right)
$$

où $E_{\ell}=\pi^{-1}\left(p_{\ell-1}\right)$. Puisque $\mu\left(\Gamma_{\ell}\right)=0$, la tangente $\tau\left(\Gamma_{\ell}\right)$ est tangente en $p_{\ell}$ à $E_{\ell}$. Pour tout indice $j \geq \ell$ on a d'après l'assertion

$$
\operatorname{Spec}\left(D X_{\ell}\left(p_{\ell}\right)\right)=\operatorname{Spec}\left(D X_{j}\left(p_{j}\right)\right)=\left\{\mu\left(\Gamma_{\ell-1}\right)\right\} \cup \operatorname{Spec}\left(\left.D X_{j}\left(p_{j}\right)\right|_{F_{j}}\right)
$$


où $F_{j}$ est le transformé strict de $E_{\ell}$ par $\pi_{j} \circ \pi_{j-1} \circ \ldots \circ \pi_{\ell+1}$. Alors $\Gamma_{j}$ est tangent à $F_{j}$ pour $j \geq \ell$ et ainsi $\Gamma_{\ell}$ est contenu dans $E_{\ell}$. Ce qui est absurde.

2.2. Foyer monodromique. Supposons $\operatorname{dim} M=2$. On dit que $p$ est un foyer monodromique contractant de $X$ s'il existe une demi-branche analytique $\Gamma=$ $c(] 0, \varepsilon[)$, où $c$ est une application analytique avec $c(0)=p$, telle que, toute orbite positive $\gamma: \mathbb{R}^{+} \longrightarrow M$ de $X$ issue d'un point $c(s), 0<s<\varepsilon$, recoupe $\Gamma$ une première fois en un point $c\left(s_{1}\right)$ avec $s_{1}<s$. L'application $c(s) \mapsto c\left(s_{1}\right)$ est l'application premier retour de Poincaré de $p$ évaluée sur $\Gamma$ pour $X$. Le résultat suivant est bien connu. Ses preuves classiques reposent sur le théorème de désingularisation des champs de vecteurs en dimension 2 [Se] ou encore sur un argument de Khovanski $[\mathrm{Kh}]$. Celle que nous proposons utilise de façon essentielle la proposition du paragraphe 1.2 .

Théorème. Supposons que $p$ soit un point singulier de $X$ et soit $\gamma$ une demicourbe intégrale de $X$ telle que $\omega(\gamma)=p$. Les propriétés suivantes sont équivalentes: i) $\gamma$ ne possède pas de tangente en $p$.

ii) $\gamma$ ne possède pas la propriété des tangentes itérées en $p$.

iii) $\gamma$ est oscillante en p par rapport à une demi-branche analytique $\Gamma$.

iv) $\gamma$ spirale autour de $p$.

v) $p$ est un foyer monodromique contractant.

vi) Il existe un voisinage $V$ (semi-analytique) de $p$ positivement invariant par $X$ tel que toute demi-courbe intégrale de $X$ issue d'un point de $V$ spirale autour de $p$.

Preuve. Il est clair que vi) implique i), que i) implique ii) et ii) implique iii) d'après la proposition de 1.2. Prouvons que iii) implique iv). Soit $c$ une application analytique de $\mathbb{R}$ dans $M$ telle que $c(0)=p, c(] 0, \varepsilon[)=\Gamma$. L'ensemble des $s>0$ tels que $X(c(s))$ et $c^{\prime}(s)$ soient colinéaires est un sous-ensemble analytique strict de $\mathbb{R}$ puisque $|\gamma| \not \subset \Gamma$. On peut le supposer vide en prenant $\varepsilon>0$ assez petit. En particulier, $\Gamma$ est transversalement orientée par $\gamma$, ce qui prouve iv). Montrons que iv) (ou iii) implique v). On sait déjà que toutes les demi-courbes intégrales de $X$ coupent $\Gamma$ transversalement et toujours dans le même sens. Soit $q_{0}=c\left(s_{0}\right)$, $q_{1}=c\left(s_{1}\right)$ deux points d'intersection consécutifs de $|\gamma|$ avec $\Gamma$ et soit $K$ le compact de bord la réunion des $\operatorname{arcs} q_{0} q_{1}$ sur $\Gamma$ et $|\gamma|$. L'argument classique utilisé dans la preuve du théorème de Poincaré-Bendixson appliqué à $K$ montre que toute demi-courbe intégrale de $X$ issue d'un point $c\left(s_{0}^{\prime}\right)$ de $\Gamma$ avec $s_{0}^{\prime}<s_{0}$ recoupe $\Gamma$ en un point $s_{1}^{\prime}$ avec $s_{1}^{\prime}<s_{1}$ (figure 4). Ceci prouve clairement les assertions v) et vi) en prenant $V=\operatorname{int} K$.

2.3. Tangentes itérées, oscillation et spiralement axial. Dans tout ce paragraphe on suppose $\operatorname{dim} M=3$ et $\gamma$ désigne une demi-courbe intégrale d'un champ de vecteurs analytique $X$ sur $M$ telle que $\omega(\gamma)=p$ est un point singulier (non nécessairement isolé) de $X$. Nous avons vu dans le paragraphe précédent 

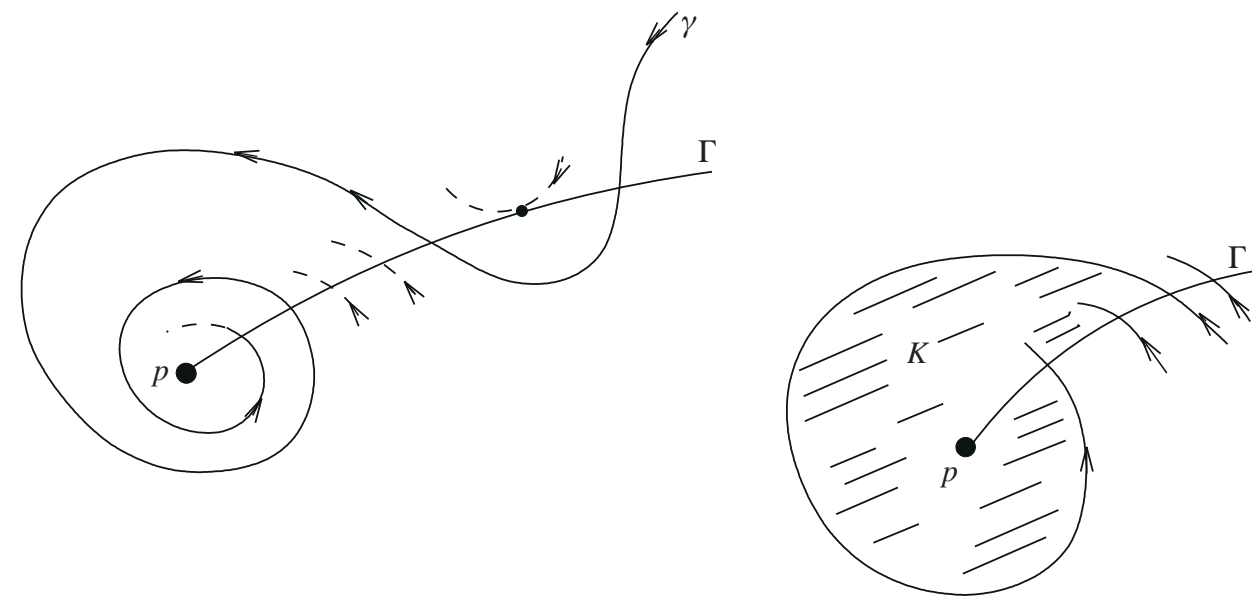

Figure 4 .

qu'en dimension 2 les propriétés $\gamma$ "oscille", " $\gamma$ possède des tangentes itérées" sont incompatibles. Cette dichotomie est propre à la dimension 2. En dimension 3, il faut la remplacer par le spiralement axial comme le montre le théorème suivant.

Théorème 1. Soit $\gamma$ une courbe intégrale d'un champ de vecteurs analytique sur une variété de dimension 3. Alors $\gamma$ spirale autour d'une demi-branche analytique $\Gamma$ en $p$ si et seulement si $\gamma$ a la propriété des tangentes itérées en $p$ et est oscillante en $p$ par rapport à une surface semi-analytique $S$.

Preuve. Si $\Gamma$ est un axe de spiralement pour $\gamma$, nous avons déjà vu avec la proposition de 1.4 que $\gamma$ a un contact plat avec $\Gamma$. De plus, $\gamma$ est oscillante par rapport à toute surface semi-analytique $S$ telle que $\Gamma \subset \overline{S \backslash \Gamma}$ d'après le lemme de 1.4 et la définition de spiralement axial. Supposons que $\gamma$ possède des tangentes itérées orientées $\mathrm{TI}^{+}(\gamma)$ et que $\gamma$ est oscillante par rapport à une surface semi-analytique $S$. Montrons que $\gamma$ spirale autour d'une demi-branche $\Gamma$.

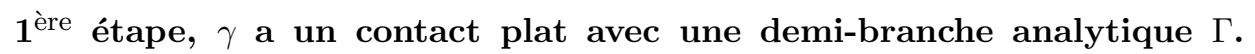
Quitte à compléter $S$ en une surface analytique et à prendre une composante irréductible, nous pouvons supposer que $S$ est irréductible et qu'il existe une carte affine $(U,(x, y, z))$ centrée en $p=\omega(\gamma)$ et une fonction analytique $f$ sur $U$ telle que

$$
\text { Sing } f \subset\{f=0\}, \operatorname{dim}(\operatorname{Sing} f) \leq 1, \quad S=U \cap\{f=0\} .
$$

De plus quitte à éclater une fois, on peut aussi supposer que $|\gamma| \subset U^{+}=\{z>0\}$. Remarquons tout d'abord que $\gamma$ coupe une infinité de fois $S^{\prime}=S \backslash \operatorname{Sing} f$. En effet, si ce n'est pas le cas, $\gamma$ coupe une infinité de fois une demi-branche analytique $\Gamma$ en $p$ contenue dans $S \cap \operatorname{Sing} f$. Les courbes $\gamma$ et $\Gamma$ ont les mêmes tangentes itérées. 
D'après la proposition 2.1, $\Gamma$ est une demi-courbe intégrale de $X$. Ainsi $|\gamma|$ est contenue dans $S$ et $\gamma$ n'est pas oscillante par rapport à $S$.

Soit $Z=S \cap\{d f(X)=0\}$ l'ensemble des points de "tangence généralisés" de $X$ et de $S$. Montrons tout d'abord que $\operatorname{dim} Z \leq 1$. En effet, $S$ étant irréductible, si $\operatorname{dim} Z=2$ on a $Z=S$. La partie lisse $S^{\prime}$ de $S$ est invariante par $X$. Puisque $\gamma$ coupe $S^{\prime},|\gamma|$ est contenu dans $S$ et ainsi $\gamma$ n'est pas oscillante par rapport à $S$. Quitte à prendre une nouvelle carte affine centrée en $p$ on peut supposer que $Z \cap U^{+}=Z^{+}$est une union finie, éventuellement vide, de demi-branches analytiques. Montrons qu'une de ces branches a un contact plat avec $\gamma$. Si ce n'est pas le cas et si $T I(\gamma)=\left\{p_{n}\right\}$ il existe $\ell$ tel que $p_{\ell}$ n'appartienne pas à la réunion des tangentes itérées de ces branches. Soit $\pi=\pi_{1} \circ \pi_{2} \circ \cdots \circ \pi_{\ell}$ le composé de la suite d'éclatements de centres $p, p_{1}, \cdots, p_{\ell-1}$. Il existe une carte affine $\left(U_{\ell}\right)$, centrée en $p_{\ell}$ telle que si $U_{\ell}^{+}$est la composante connexe de $U_{\ell} \backslash \pi^{-1}(p)$ contenant $\gamma_{\ell}=\pi^{-1}(\gamma)$ on ait $Z_{\ell}^{+}=U_{\ell}^{+} \cap \pi^{-1}(Z)=\emptyset$. Posons $S_{\ell}=\pi^{-1}(S), f_{\ell}=f \circ \pi$ et soit $X_{\ell}$ le relevé de $X$ par $\pi$. La restriction de $\pi$ à $U_{\ell}^{+}$étant un isomorphisme sur son image, on a:

$$
S_{\ell} \cap\left\{d f_{\ell}\left(X_{\ell}\right)=0\right\} \cap U_{\ell}^{+}=Z_{\ell}^{+}=\emptyset .
$$

Ainsi, en oubliant l'indice $\ell$ et en identifiant une carte affine avec $\mathbb{R}^{3}$, nous sommes dans la situation suivante: $f$ est une fonction analytique sur $\mathbb{R}^{3}, X$ un champ de vecteurs analytique sur $\mathbb{R}^{3}, \gamma$ une demi-courbe intégrale de $X$ avec $\omega(\gamma)=\{0\}$ et nous devons prouver que les deux conditions:

$$
\# f^{-1}(0) \cap|\gamma| \cap U^{+}=\infty \quad, \quad\{d f(X)=0\} \cap f^{-1}(0) \cap U^{+}=\emptyset
$$

sont incompatibles, où $U^{+}$est une composante connexe du complémentaire de $x^{\varepsilon_{1}} y^{\varepsilon_{2}} z=0$ avec $\varepsilon_{1}, \varepsilon_{2}$ égaux à 0 ou 1 telle que $|\gamma| \subset U^{+}$. Soit $S_{1}$ une composante connexe de $f^{-1}(0) \cap U^{+}$qui est coupée une infinité de fois par $\gamma$. D'après la deuxième condition, $S_{1}$ est une hypersurface fermée de $U^{+}$et $U^{+} \backslash S_{1}$ a deux composantes connexes. Puisque $\left.d f(X)\right|_{S_{1}} \neq 0$ toute demi-orbite positive de $X$ issue d'un point de $S_{1}$ et contenue dans $U^{+}$est contenue dans une de ces deux composantes connexes; ceci contredit la condition \# $|\gamma| \cap S^{+}=\infty$.

2. Fin de la preuve. D'après les arguments développés dans la première partie, l'ensemble $Z^{+}$des points de tangence de $X$ avec $S^{+}=f^{-1}(0) \cap\{z>0\}$ est la réunion de $\Gamma$, une demi-branche analytique ayant un contact plat avec $\gamma$, et d'un nombre fini de demi-branches analytiques parasites. D'après le lemme de 1.4, et en repprenant ses notations, il existe $n_{0}$ tel que, pour $n \geq n_{0}, W_{n} \cap Z^{+}=\Gamma$ et, $S_{1}, S_{2}, \cdots, S_{r}$ désignant les composantes connexes de $S^{+} \cap W_{n} \backslash \Gamma$, les couples $\left(W_{n} \cap S_{i}, W_{n}\right)$ sont des triangles de côté $\Gamma$. Ainsi, il existe $\ell_{0}$ tel que, pour $n \geq n_{0}$, $R_{n}=W_{n} \cap S_{\ell_{0}}$ soit transversalement orienté par $\gamma$. De plus, puisque $\gamma$ a un contact plat avec $\Gamma$, on sait que $\gamma(t) \in W_{n}$ pour $t$ assez grand. Nous avons ainsi déjà montré que les triangles $\left(R_{n}, W_{n}\right)$, pour $n \geq n_{0}$, satisfont aux conditions de la définition " $\gamma$ spirale autour de $\Gamma$ ". 

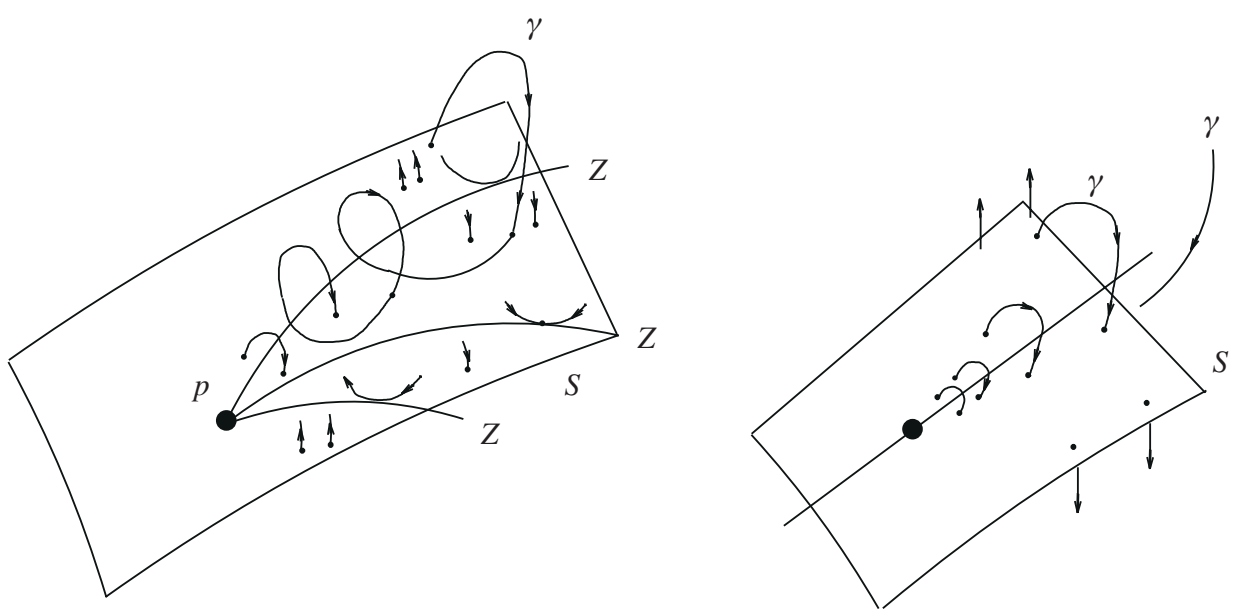

Figure 5 .

Montrons que ces conditions sont vérifiées pour tout triangle de côté $\Gamma$. Soit $(T, W)$ un tel triangle. D'après le lemme de 1.4 , il existe $n_{1}$ et une composante connexe $T_{1}$ de $W_{n_{1}} \cap T$ telle que pour $n>n_{1}$ le couple $\left(T_{1} \cap W_{n}, W_{n}\right)$ soit un triangle de côté $\Gamma$ compatible avec $\left(R_{n}, W_{n}\right)$. L'ensemble des points de tangence de $X$ avec $T_{1}$ est d'après les arguments développés dans la première partie, un ensemble semi-analytique $Z_{1}$ de dimension au plus 1 . D'après l'assertion ii) du lemme de 1.4, il existe $n_{2}$ tel que, pour $n>n_{2}$, on ait $Z_{1} \cap W_{n_{1}}=\emptyset$; c'est-à-dire que $T_{1} \cap W_{n_{1}}$ est transverse à $X$.

Montrons, pour achever la preuve, que $\gamma$ coupe une infinité de fois $T_{1} \cap W_{n}$ pour $n>n_{2}$ lorsque $T_{1} \cap W_{n}$ est différent de $R_{n}$. Soient $\gamma\left(t_{0}\right), \gamma\left(t_{1}\right)$ avec $t_{0}<$ $t_{1}$ deux points d'intersection consécutifs de $\gamma$ avec $R_{n}$. Les triangles $\left(R_{n}, W_{n}\right)$, $\left(T_{1} \cap W_{n}, W_{n}\right)$ étant compatibles, le complémentaire de $T_{1} \cup \Gamma$ dans $W_{n} \backslash R_{n}$ a deux composantes connexes. Puisque $\gamma\left(\left[t_{0}, t_{1}\right]\right)$ relie deux composantes connexes distinctes du bord de $W_{n} \backslash R_{n}$, il existe $t_{0}<t_{2}<t_{1}$ tel que $\gamma\left(t_{2}\right)$ appartient à $T_{1} \cap W_{n}$.

Corollaire 1. La propriété de spiralement axial est invariante par éclatement et effondrement pour les courbes intégrales d'un champ de vecteurs analytique réel.

Preuve. Soit $\pi: M_{1} \longrightarrow M$ l'éclatement de centre $p \in M$ et soit $\gamma$ une courbe analytique de $X$ tel que $\omega(\gamma)=p$. Supposons que $\gamma$ spirale autour d'une demibranche $\Gamma$. Alors $\gamma$ possède des tangentes itérées et oscille par rapport à une surface $S$. La courbe $\gamma_{1}=\pi_{1}^{-1} \circ \gamma$ possède des tangentes itérées et oscille par rapport à $S_{1}=\pi_{1}^{-1}(S)$. D'après le théorème, $\gamma_{1}$ spirale autour d'une demi-branche analytique.

Supposons que $\gamma_{1}=\pi \circ \gamma$ spirale autour de $\Gamma_{1}$. Alors $\pi\left(\Gamma_{1}\right)=\Gamma$ est une 
demi-branche analytique qui a un contact plat avec $\gamma$. Si $S$ est une surface semianalytique telle que $\Gamma \subset \overline{S \backslash \Gamma}$, d'après la définition du spiralement on sait que:

$$
\# \quad \pi^{-1}(S) \cap\left|\gamma_{1}\right|=\# \quad S \cap|\gamma|=\infty .
$$

Ainsi $\gamma$ spirale autour de $\Gamma$ d'après le théorème.

Corollaire 2. (Propriété caractéristique du spiralement axial). Soit $\gamma$ une courbe intégrale de $X$ telle $\omega(\gamma)=p$ et soit $\Gamma$ une demi-branche analytique en $p$. Alors $\gamma$ spirale autour de $\Gamma$ si et seulement si pour toute surface semi-analytique $S$ de $M^{3}$ on a l'équivalence suivante:\# $S \cap|\gamma|=\infty \Longleftrightarrow \Gamma \subset \overline{S \backslash \Gamma}$.

Preuve. Supposons que $\gamma$ spirale autour de $\Gamma$ et soit $S$ une surface. Si $\Gamma \subset$ $\overline{S \backslash \Gamma}$, d'après le lemme de 1.4 (existence de triangle analytique) et la définition du spiralement $\gamma$ est oscillante par rapport à $S$. Si $\Gamma \cap \overline{S \backslash \Gamma}=\emptyset$, d'après le lemme 1.4, il existe un voisinage ouvert $W_{n}$ de $\Gamma$ tel que $S \cap W_{n}=\emptyset$ et $\gamma(t) \in W_{n}$ pour $t$ assez grand.

Supposons que l'équivalence soit vraie pour tout $S$ de $M^{3}$. L'argument utilisé ci-dessus montre que l'implication $\# S \cap|\gamma|=\infty \Longrightarrow \Gamma \subset \overline{S \backslash \Gamma}$ entraîne que $\gamma$ a un contact plat avec $\Gamma$. La deuxième implication entraîne que $\gamma$ est oscillante. Ainsi, $\gamma$ spirale autour de $\Gamma$ d'après le théorème.

Corollaire 3. Supposons que $\gamma$ (courbe intégrale de $X$ sur $M^{3}$ ) ait un contact plat avec une demi-branche $\Gamma$ lisse et soit $\left(x_{1}, x_{2}, z\right)$ une carte affine en $p=\omega(\gamma)$ telle que:

$$
\Gamma=\left\{x_{1}=x_{2}=0, z>0\right\} \quad, \quad \gamma(t)=\left(x_{1}(t), x_{2}(t), z(t)\right) .
$$

Alors $\gamma$ spirale autour de $\Gamma$ si et seulement si la projection $\gamma_{0}(t)=\left(x_{1}(t), x_{2}(t)\right)$ de $\gamma$ sur $z=0$ spirale autour de $p$.

Preuve. On a déjà vu dans 1.4 que si $\gamma$ spirale autour de $\Gamma$, alors $\gamma_{0}$ spirale autour de $p$. Si $\gamma_{0}$ spirale autour de $p$, il est clair que $\gamma$ est oscillante par rapport au plan $x_{1}=0$ et ainsi $\gamma$ spirale autour de $\Gamma$ d'après le théorème.

Remarque. Ce corollaire nous incite à comparer le spiralement autour d'un point en dimension 2 et le spiralement axial en dimension 3. Nous avons vu (avec le théorème de 2.2) que dans le premier cas, l'existence d'une courbe intégrale qui spirale autour de $p$ implique que $p$ est un foyer monodromique, c'est-à-dire l'existence d'un voisinage (semi-analytique) de $p$, invariant par $X$ et formé de courbes intégrales qui spiralent autour de $p$. En dimension 3, nous savons prouver ce résultat lorsque l'axe de spiralement n'est pas formé de points fixes. C'est l'objet essentiel du chapitre suivant. 


\section{Axes de tourbillonnement}

Soient $M$ une variété analytique réelle de dimension trois et $X$ un champ de vecteurs analytique sur $M$.

Définitions. Si $\Gamma$ est une demi-branche analytique en un point $p$ de $M$ nous dirons que:

i) $\Gamma$ est un axe de spiralement non dégénéré de $X$ si $\Gamma$ est un axe de spiralement pour une demi-courbe intégrale $\gamma$ et $\Gamma$ n'est pas contenu dans Sing $X$.

ii) $\Gamma$ est un axe de tourbillonnement de $X$ s'il existe un voisinage ouvert semi analytique $V$ de $\Gamma$ qui est positivement invariant par $X$ et tel que $\Gamma$ soit un axe de spiralement pour toute demi-courbe intégrale positive $\gamma$ d'origine un point de $V \backslash \Gamma$. Nous dirons alors que $V$ est un domaine de tourbillonnement pour $\Gamma$. De plus nous dirons que $\Gamma$ est un axe de tourbillonnement non dégénéré si $\Gamma \not \subset$ Sing $X$.

Le but principal de ce chapitre est de montrer le théorème suivant.

Théorème 2. Si $\Gamma$ est un axe de spiralement non dégénéré de $X$ alors $\Gamma$ est un axe de tourbillonnement de $X$.

Les axes de tourbillonnement non dégénérés sont clairement des "centres organisateurs" de la dynamique au sens de $\mathrm{R}$. Thom $\left[\mathrm{Th}_{2}\right]$. Le résultat suivant montre que ces axes possèdent les propriétés de finitude, d'analyticité que l'on souhaite.

Corollaire. Soit $p$ un point de M. L'ensemble des axes de spiralement en $p$ est fini. L'ensemble des axes de tourbillonnement non dégénérés est localement fini en $p$, c'est-à-dire qu'il existe un voisinage de $p$ qui ne contient qu'un nombre fini d'axes de tourbillonnement non dégénérés.

On déduit de ce corollaire que l'ensemble des axes de spiralement de $X$ est contenu dans un sous-ensemble semi analytique de dimension 1. Ces résultats ne sont pas entièrement satisfaisants. Nous ne savons pas si le nombre d'axes de tourbillonnement (éventuellement dégénérés) est fini. En particulier, nous ne savons pas si la situation suivante peut se présenter pour un champ de vecteurs $X$ analytique dans le voisinage de $0 \in \mathbb{R}^{3}$ : la droite $\Delta=\{y=z=0\}$ est contenue dans Sing $X$ et il existe une suite de points $p_{n}=\left(x_{n}, 0,0\right), x_{n+1}<x_{n}$, tendant vers 0 , tel que le germe de $\Delta$ en $p_{n}$ soit un axe de tourbillonnement de $X$ (figure 6 ).

La preuve du théorème 2 est décomposée en trois étapes. Dans la première nous étudions le spectre de $D X(p)$ lorsque $p$ est un point singulier élémentaire de $X$, i.e. lorsque $D X(p)$ n'est pas nilpotente et nous en déduisons le concept de singularité réduite spiralante. Dans le paragraphe suivant nous montrons que le théorème est vrai pour ces singularités. Enfin, dans la dernière étape, nous montrons tout d'abord qu'il est vrai pour les singularités élémentaires et ensuite en utilisant des éclatements et des ramifications nous montrons que le cas général 

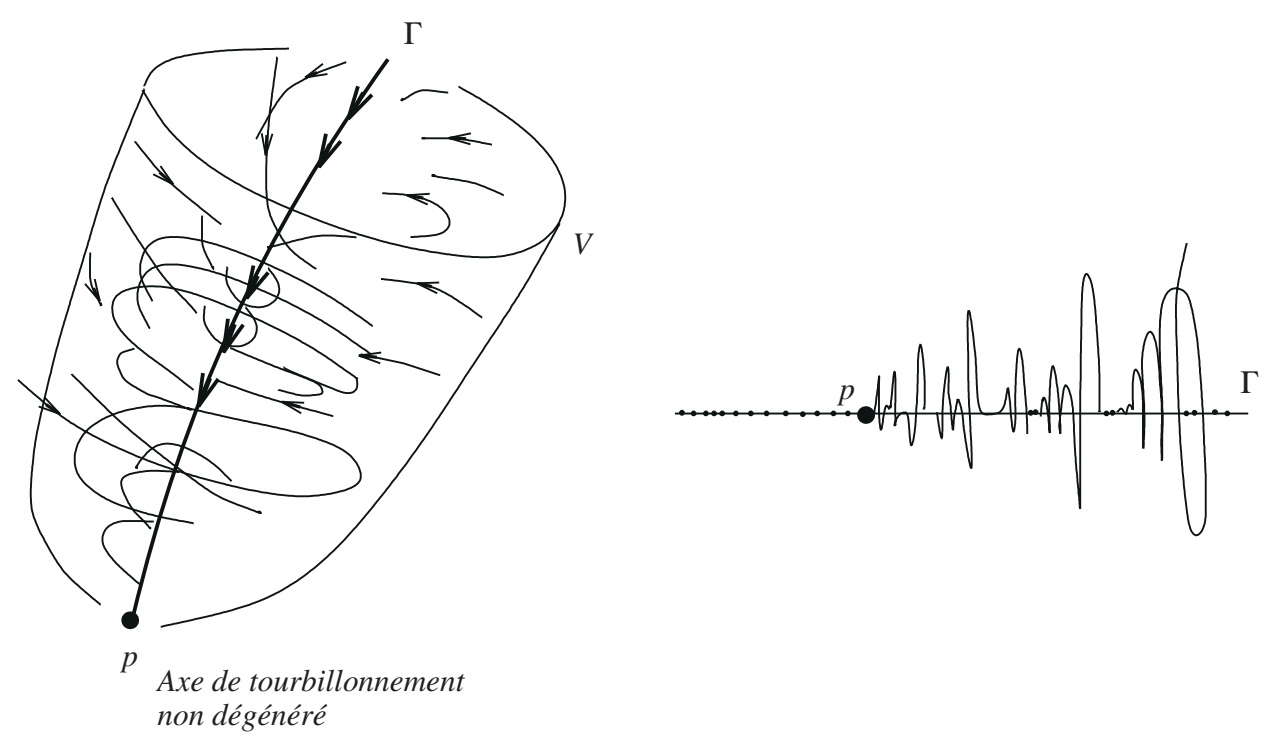

Figure 6 .

se ramène au cas élémentaire. Le paragraphe suivant est consacré à la preuve du résultat de finitude, elle repose sur l'uniformisation locale des champs de vecteurs en dimension 3. Enfin, dans le dernier paragraphe nous étudierons le caractère "équilibré" des axes de tourbillonnement non dégénérés.

1. Singularités élémentaires. La preuve de la proposition suivante utilise essentiellement la proposition 2.1.2. du chapitre précédent.

Proposition. Soit $p$ une singularité élémentaire $(D X(p)$ non nilpotente) d'un champ de vecteurs analytique $X$ sur $M$. Supposons que $\Gamma$ soit un axe de spiralement non dégénéré d'une demi-courbe intégrale $\gamma$ de $X$ avec $p=\omega(\gamma)$. Alors $\Gamma$ est lisse, sa tangente en $p$ est une direction propre de $D X(p)$ de valeur propre $\mu(\Gamma)=0$ et les deux autres valeurs propres de $D X(p)$ sont conjuguées à partie réelle négative ou nulle.

Preuve. D'après le théorème $1, \gamma$ a un contact plat avec $\Gamma$ et, d'après la proposition 2.1.2., la tangente à $\Gamma$ en $p$ est une direction propre de $D X(p)$ de valeur propre $\mu(\Gamma)=0$. Notons $\pi_{k}: M_{k} \rightarrow M_{k-1}$ la suite d'éclatements de centre les points $\left\{p_{k}\right\}$ de $T I(\Gamma)$ avec $p_{0}=p, M_{0}=M$ et soit $\ell \geq 0$ le premier entier tel que $\Gamma_{\ell}=\pi_{\ell}^{-1}\left(\Gamma_{\ell-1}\right)$ soit lisse. D'après l'assertion de la preuve de proposition 2.1.2.

$$
\operatorname{Spec}(D X(p))=\operatorname{Spec}\left(D X_{\ell}\left(p_{\ell}\right)\right) \text {. }
$$

Ainsi, pour étudier le spectre de $D X(p)$, nous pouvons supposer que $\Gamma$ est lisse. Choisissons une carte affine en $p$ telle que $\Gamma=\{x=y=0, z>0\}$. Soit $\gamma^{\prime}$ l'image 
de $\gamma$ par la projection $(x, y, z) \mapsto(x, y)$. D'après I.1.4, la demi-courbe $\gamma^{\prime}$ spirale autour de $p$ dans le plan $z=0$. Soit $\sigma: \widetilde{M} \rightarrow M$ l'éclatement de centre la droite $x=y=0$ et soit $\widetilde{\gamma}=\sigma^{-1} \circ \gamma$ le relevé de $\gamma$ par $\sigma$. Puisque $\gamma^{\prime}$ spirale autour de $p$ on a $\omega(\widetilde{\gamma})=\sigma^{-1}(p) \simeq S^{1}$. Montrons (par l'absurde) que les valeurs propres $\mu^{\prime}$, $\mu^{\prime \prime}$ de $D X(p)$ distinctes de $\mu(\Gamma)$ sont conjuguées, en particulier $\mu^{\prime}=\bar{\mu}^{\prime \prime} \neq 0$. Si ce n'est pas le cas, $\mu^{\prime}, \mu^{\prime \prime}$ sont réelles distinctes. On peut choisir les coordonnées $x$, $y$, telles que le jet d'ordre 1 de $X$ en $p$ s'écrive (compte tenu du fait que $x=y=0$ est invariant)

$$
J^{1} X(p)=\mu^{\prime} x \frac{\partial}{\partial x}+\mu^{\prime \prime} y \frac{\partial}{\partial y}, \quad \text { avec } \mu^{\prime} \neq 0
$$

Soient $(\widetilde{x}, \widetilde{y}, \widetilde{z})$ des coordonnées sur $\widetilde{M}$ au point $\widetilde{p} \in \mathbb{R} P(1)$ correspondant à $y=z=0$ telles que $\widetilde{x}=x / y, \widetilde{y}=y, \widetilde{z}=z$, et soit $\widetilde{X}$ le relevé de $X$ par $\sigma$. Alors la restriction de $\widetilde{X}$ à $\sigma^{-1}(p)$ s'écrit dans ces coordonnées

$$
\left.\widetilde{X}\right|_{\sigma^{-1}(p)}=\left(\mu^{\prime}-\mu^{\prime \prime}\right) \widetilde{x} \frac{\partial}{\partial \widetilde{x}} .
$$

Puisque $\mu^{\prime} \neq \mu^{\prime \prime}, \widetilde{p}$ est un point singulier hyperbolique de $\left.\widetilde{X}\right|_{\sigma^{-1}(p)}$ et alors $\sigma^{-1}(p)$ ne peut pas être un cycle limite de $\widetilde{\gamma}$, en particulier $\omega(\widetilde{\gamma}) \neq \sigma^{-1}(p)$.

Montrons que $\Gamma$ est lisse. Si ce n'est pas le cas il existe un entier $k>0$ tel que $\Gamma_{k}$ est tangente au diviseur exceptionnel $\pi_{k-1}^{-1}\left(p_{k}\right)=E_{k}$. Ainsi $\mu\left(\Gamma_{k}\right)=0$ est une valeur propre de la restriction de $D X_{k}\left(p_{k}\right)$ à $E_{k}$. D'après l'assertion de la preuve de la proposition 2.1.2., ceci est impossible puisque l'on a:

$\operatorname{Spec}\left(\left.D X_{k}\left(p_{k}\right)\right|_{E_{k}}\right)=\operatorname{Spec}\left(\left.D X_{k-1}\left(p_{k-1}\right)\right|_{E_{k-1}}\right)=\cdots=\left\{\mu^{\prime}, \bar{\mu}^{\prime}\right\}$.

Corollaire. Soit p une singularité élémentaire de X. Il existe au plus deux axes de spiralement non dégénérés en $p$. De plus, il n'existe pas de suite de points $\left\{q_{n}\right\}, q_{n} \neq p$, tendant vers $p$ telle que $X$ possède un axe de spiralement non dégénéré $\Gamma_{n}$ au point $p_{n}$.

Preuve. Soit $\Gamma$ un axe de spiralement en $p$ pour $\gamma$. La suite de points $T I(\Gamma)=\left\{p_{k}\right\}$ est uniquement déterminée par la condition: le point $p_{k} \in \mathbb{R} P(2)=\pi_{k-1}^{-1}\left(p_{k-1}\right)$ est l'image par la projection canonique de $\mathbb{R}^{3}$ sur $\mathbb{R} P(2)$ du noyau de $D X_{k-1}\left(p_{k-1}\right)$. Ceci montre la première partie du corollaire. Pour prouver la seconde partie, raisonnons par l'absurde. Supposons qu'il existe une suite $\left\{q_{n}\right\} \rightarrow p$ et une double suite $\left\{\left(\Gamma_{n}, \gamma_{n}\right)\right\}$ d'axes de spiralement $\Gamma_{n}$ pour des courbes intégrales $\gamma_{n}$ de $X$ aux points $q_{n}$. Puisque les courbes $\Gamma_{n}, \gamma_{n}$ ne rencontrent pas le lieu singulier de $X$, on peut supposer (quitte à diviser $X$ par une unité) que $\operatorname{dim} \operatorname{Sing} X=1$. Il existe une demi-branche $\Delta \subset$ Sing $X$ contenant une infinité de $q_{n}$. Puisque Ker $D X(p)$ est de dimension 1 , on peut choisir $q_{n} \in \Delta$ voisin de $p$ tel que Ker $D X\left(q_{n}\right)$ soit aussi de dimension 1 et tel que le germe $\Delta_{n}$ de $\Delta$ en $q_{n}$ soit non singulier. Puisque $T_{q_{n}}$ $\Delta_{n}$ et $T_{q_{n}} \Gamma_{n}$ sont contenus dans Ker $D X\left(q_{n}\right)$ on a $T_{q_{n}} \Delta_{n}=T_{q_{n}} \Gamma_{n}$. En répétant 
cet argument après éclatement on a $T I\left(\Delta_{n}\right)=T I\left(\Gamma_{n}\right)$. Ainsi $\Gamma_{n} \equiv \Delta_{n} \subset$ Sing $X$ ce qui contredit l'hypothèse $\Gamma_{n}$ est un axe de spiralement non dégénéré.

2. Singularités spiralantes réduites. Dans le paragraphe suivant nous verrons qu'après un nombre fini d'éclatements, les singularités élémentaires qui portent des axes de spiralement non dégénérés satisfont à la définition suivante.

Définition. Un point $p$ de Sing $X$ est une singularité spiralante réduite s'il existe une carte affine $((x, y, z)), U)$ en $p$ telle que $\Gamma=\{x=y=0, z>0\}$ soit un axe de spiralement d'une demi-courbe intégrale $\gamma_{0}$ et telle que

$$
X=\sum_{i=0}^{q} z^{i} L_{i}+z^{q+1}\left(-\frac{\partial}{\partial z}+V\right) \quad \text { ò̀ } q \geq 1 \text { et }
$$

i) $L_{i}(x, y)=\left(\alpha_{i} x+\beta_{i} y\right) \frac{\partial}{\partial x}+\left(\gamma_{i} x+\delta_{i} y\right) \frac{\partial}{\partial y}$ avec $L_{0}$ non nilpotent.

ii) $V=a(x, y, z) \frac{\partial}{\partial x}+b(x, y, z) \frac{\partial}{\partial y}$ avec $a(0,0, z)=b(0,0, z) \equiv 0$.

De telles coordonnées s'appellent des coordonnées adaptées à $(X, p)$.

La composante de $X$ sur $\frac{\partial}{\partial z}$ étant $-z^{q+1}$ les demi-courbes intégrales $\gamma$ de $X$ d'origine un point $\gamma(0) \in\{z>0\}$ sont contenues dans $\{z>0\}$ et peuvent être paramétrées par $z$. Plus précisément, ces courbes sont solutions de l'équation différentielle dans $\mathbb{R}^{2}$ (dépendant du temps $z$ ) suivante:

$$
\frac{d u}{d z}=-\frac{1}{z^{q+1}}\left(\sum_{i=0}^{q} z^{i} L_{i}(u)\right)-V(u, z) \quad \text { avec } \quad u=(x, y) .
$$

On écrit $\underline{\gamma}(z)=(x(z), y(z), z)=(u(z), z)$ la paramétrisation correspondante de $|\gamma|$ et on pose $r^{2}(z)=\|u(z)\|^{2}=x^{2}(z)+y^{2}(z)$. Compte tenu de la condition $V(0, z) \equiv 0$, on peut supposer que $\|V(u, z)\|<1$ pour $z \in C_{1}$ avec $C_{\varepsilon}=\left\{x^{2}+y^{2}<\right.$ $1,0<z<\varepsilon\}$.

D'après la proposition du paragraphe précédent, le spectre de $D X(p)=L_{0}$ est du type $\{0, \lambda, \bar{\lambda}\}$. On dit que $(X, p)$ est asymptotiquement monodromique (resp. non asymptotiquement monodromique) si $\lambda \neq \bar{\lambda}$ (resp. $\lambda=\bar{\lambda}$ ). Pour prouver le théorème pour les singularités réduites nous envisageons ces deux cas. Le premier a déjà été étudié dans [Bo-Du] avec une autre approche.

Proposition 2.1. Soit $(X, p)$ une singularité spiralante réduite, asymptotiquement monodromique d'axe $\Gamma$. Alors $\Gamma$ est un axe de tourbillonnement. De plus dans des coordonnées adaptées bien choisies $X$ est transverse aux niveaux des fonctions $z, x^{2}+y^{2}$ et $y / x$ sur le cylindre $C_{\varepsilon} \backslash \Gamma$. 
Preuve. Un petit calcul (voir encore [Tak]) montre qu'il existe un changement de coordonnées dépendant de $z$ du type

$$
u=(x, y) \mapsto\left(A_{0}+z A_{1}+\cdots+z^{q} A_{q}\right)(u), \quad A_{i} \in \mathcal{M}_{2,2}(\mathbb{R})
$$

qui permet d'écrire les $L_{i}$ pour $i=0,1,2, \ldots, q$, sous la forme:

$$
L_{i}(x, y)=\left(\alpha_{i} x-\beta_{i} y\right) \frac{\partial}{\partial x}+\left(\beta_{i} x+\alpha_{i} y\right) \frac{\partial}{\partial y} \quad, \quad \text { avec } \quad \beta_{0} \neq 0 .
$$

Dans ces coordonnées on peut supposer (compte tenu de la condition $V(0, z) \equiv 0$ ) que:

$$
<u, \frac{d u}{d z}>=\frac{d r^{2}}{d z}=-\frac{r^{2}}{z^{q+1}}\left(\alpha_{0}+\alpha_{1} z+\cdots+\alpha_{q} z^{q}\right)+r^{2} \varphi(u, z)
$$

avec $|\varphi(u, z)|<1$ pour $(u, z) \in C_{\varepsilon}$. Montrons tout d'abord que l'hypothèse, il existe $\gamma_{0}$ ayant un contact plat avec $\{x=y=0, z>0\}$, implique l'existence de $j \in\{0,1, \cdots, q-1\}$ tel que $\alpha_{i}=0$ si $i<j$ et $\alpha_{j}<0$. Supposons que ce ne soit pas le cas et étudions l'équation différentielle vérifiée par $r_{0}^{2}(z)=x_{0}^{2}(z)+y_{0}^{2}(z)$, où $\underline{\gamma}_{0}(z)=\left(x_{0}(z), y_{0}(z), z\right)$ est une réparametrization de $\gamma_{0}$ par la hauteur $z$. On a alors trois possibilités

i) $\alpha_{0}=\alpha_{1}=\cdots \alpha_{j-1}=0, \alpha_{j}>0$ avec $j \leq q$. On a alors pour $z$ assez petit, $\frac{d r_{0}^{2}}{d z}(z)$ strictement négatif, ce qui contredit $\omega\left(\gamma_{0}\right)=0$.

ii) $\alpha_{0}=\alpha_{1}=\cdots=\alpha_{q-1}=0, \alpha_{q}<0$. Pour $z$ assez petit on a $\frac{1}{r_{0}^{2}(z)} \frac{d r_{0}^{2}(z)}{d z}>$ $\frac{a}{z}$ avec $a=-\frac{\alpha_{q}}{2}$. On en déduit $r_{0}^{2}(z)>A z^{a}$ avec $A>0$ et alors $\gamma_{0}$ n'a pas un contact plat avec $\Gamma$.

iii) $\alpha_{0}=\alpha_{1}=\cdots=\alpha_{q}=0$. On voit que $r_{0}(z)$ ne tend pas 0 si $z \rightarrow 0$.

Soit $\alpha=-\alpha_{j}>0, s=q-j \geq 1$. On peut écrire

$$
\frac{d r^{2}}{d z}=\frac{\alpha r^{2}}{z^{s+1}}(1+z \Psi(u, z))
$$

où $\Psi$ est bornée $\operatorname{sur} C_{1}$. Il existe $\varepsilon, a>0$ tels que $0<\frac{d r^{2}}{d z}<s a \frac{r^{2}}{z^{s+1}}$ si $(u, z) \in$ $C_{\varepsilon}$. Le cylindre $C_{\varepsilon}$ est positivement invariant par $X$ et pour toute demi-courbe intégrale $\underline{\gamma}(z)=(x(z), y(z), z), r^{2}(z)=x^{2}(z)+y^{2}(z)$ on a

$$
0<\frac{1}{r^{2}(z)} \frac{d r^{2}(z)}{d z}<\frac{s a}{z^{s+1}} .
$$


On en déduit $r^{2}(z)<A \exp \left(-a z^{s}\right)$. Ainsi $\omega(\gamma)=0$ et $\gamma$ a un contact plat avec $\{x=y=0, z>0\}$. Pour terminer la preuve de la proposition remarquons que l'on a

$$
\left(x^{2}+y^{2}\right)^{-1}(x d y-y d x)(X)=2 \beta_{0}(1+z f(x, y, z))
$$

où $f$ est bornée sur $C_{1}$. Ainsi pour $\varepsilon>0$ assez petit toute demi-courbe intégrale $\gamma$ d'origine un point de $C_{\varepsilon} \backslash \Gamma$ coupe transversalement les niveaux de $y / x$. De plus, l'équation différentielle ci-dessus montre que la projection de $\gamma$ sur $z=0$ spirale autour de 0 . D'après le corollaire I.3, $\Gamma$ est un axe de spiralement de $\gamma$.

Proposition 2.2. Soit $(X, p)$ une singularité spiralante réduite, non asymptotiquement monodromique d'axe $\Gamma=\{x=y=0, z>0\}$. Alors il existe $\varepsilon>0$ telle que, dans des coordonnées adaptées $(x, y, z), C_{\varepsilon}=\left\{x^{2}+y^{2}<1,0<z<\varepsilon\right\}$ soit un domaine de tourbillonnement de $X$. De plus $X$ est transverse aux niveaux des fonctions $x^{2}+y^{2}$ et $z$ sur $C_{\varepsilon} \backslash \Gamma$.

Preuve. Soit $\gamma_{0}$ la demi-courbe intégrale de $X$ qui spirale autour de $\Gamma$. Puisque $\omega\left(\gamma_{0}\right)=\{0\}$, la valeur propre $\lambda=\bar{\lambda}$ est strictement négative. Quitte à multiplier $X$ par une constante et à effectuer un changement de coordonnées linéaire en $(x, y)$ on peut écrire

$$
L_{0}=-\left(x \frac{\partial}{\partial x}+y \frac{\partial}{\partial y}\right)+\delta x \frac{\partial}{\partial y} \quad \text { avec } \quad \delta=0 \quad \text { ou } \quad \delta=1 .
$$

L'équation différentielle vérifiée par $r^{2}(z)=x^{2}(z)+y^{2}(z)$, où $\underline{\gamma}(z)=(x(z), y(z)$, $z$ ) est la réparametrization d'une courbe intégrale $\gamma$, s'écrit:

$$
\frac{d r^{2}}{d z}=\frac{r^{2}}{z^{q+1}} \varphi(x(z), y(z), z)
$$

où $0<a<\varphi(x, y, z)$ pour $(x, y, z) \in C_{\varepsilon}$. Les arguments de la preuve de la proposition précédente montrent immédiatement que $C_{\varepsilon}$ est positivement invariant par $X$, que toute demi-courbe intégrale $\gamma: t \mapsto \gamma(t)$, avec $\gamma(0) \in C_{\varepsilon}$, a un contact plat avec $\Gamma$ en 0 et que $X$ est transverse aux niveaux de $x^{2}+y^{2}$ et de $z$. Pour montrer qu'une telle demi-courbe est oscillante considérons l'éclatement polaire de $x=y=0$ avec les notations suivantes:

$$
\begin{aligned}
& \pi: S^{1} \times \mathbb{R} \times \mathbb{R} \rightarrow \mathbb{R}^{3} \quad \pi(\underline{x}, r, z)=(r \underline{x}, z), \\
& D^{*}=\pi^{-1}(\{x=y=0\}), C_{\varepsilon}^{*}=\pi^{-1}\left(C_{\varepsilon}\right), X^{*}=\pi_{*}^{-1}(X) .
\end{aligned}
$$

Le diviseur $D^{*}$ est une variété centrale globale de $X^{*}$, le relevé de $X$ par $\pi$. L'assertion suivante est plus ou moins classique.

Assertion sur la variété centrale. Soit $\gamma^{*}$ une demi-courbe intégrale positive de $X^{*}$ (maximale) d'origine $\gamma^{*}(0) \in C_{\varepsilon}^{*}$. Il existe une unique courbe intégrale 

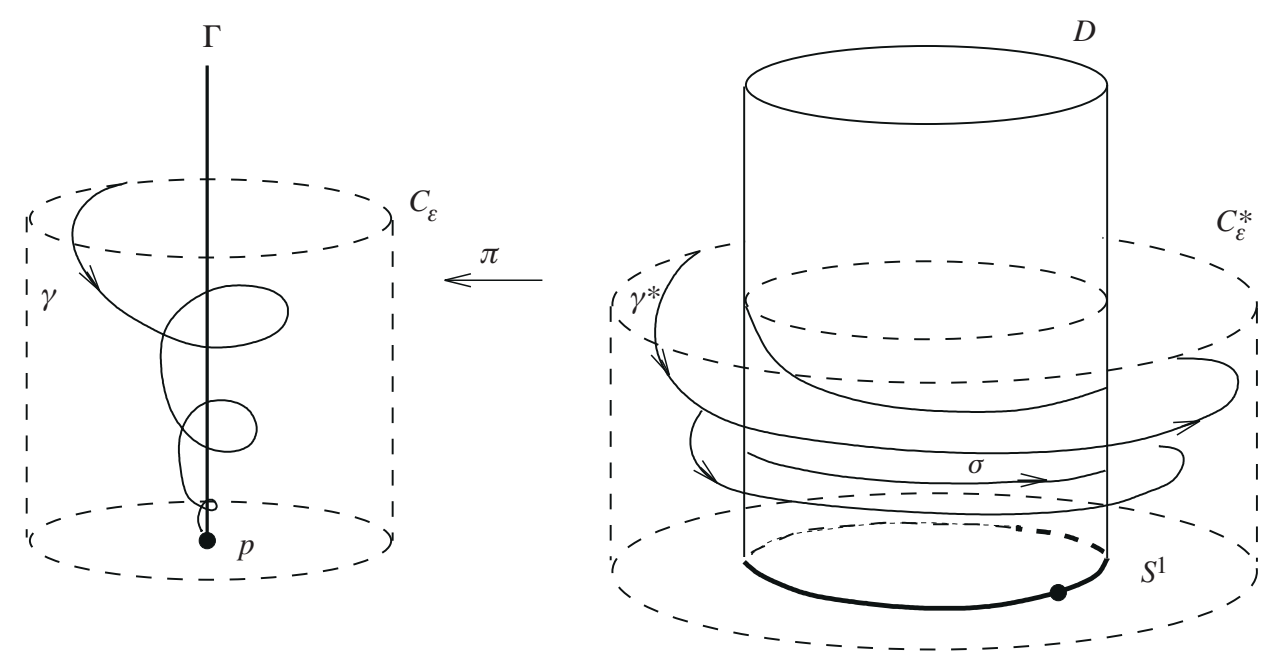

Figure 7.

maximale positive $\sigma$ de $X^{*}$ contenue dans $D^{*} \cap C_{\varepsilon}^{*}$ telle que la distance de $\gamma^{*}(t)$ $\grave{a} \sigma(t)$ soit bornée par $C \exp (-a t)$ pour $t \geq 0$ où $a, C>0$. On dit alors que $\sigma$ est la courbe accompagnatrice de $\gamma^{*}$.

Soit maintenant $\gamma_{0}^{*}=\pi^{-1} \circ \gamma_{0}$ où $\gamma_{0}$ spirale autour de $\Gamma$ et soit $\sigma_{0}$ sa courbe accompagnatrice. Un argument de la preuve de la proposition du paragraphe précédent montre que $\omega\left(\gamma_{0}^{*}\right)=\omega\left(\sigma_{0}\right)=\pi^{-1}(p) \simeq S^{1}$. Le cercle $\pi^{-1}(p)$ est un cycle limite de $\left.X^{*}\right|_{D^{*}}$ et $\omega(\sigma)=\pi^{-1}(p)$ pour toute courbe intégrale dans $C_{\varepsilon}^{*} \cap D^{*}$. Soit $\gamma$ tel que $\gamma(0) \in C_{\varepsilon}, \gamma^{*}=\pi^{-1} \circ \gamma$ et soit $\sigma$ la courbe accompagnatrice de $\gamma^{*}$. Alors, d'après l'assertion, $\omega\left(\gamma^{*}\right)=\omega(\sigma)=\pi^{-1}(p)$ et ainsi $\gamma$ est oscillante par rapport au plan $x=0$. On conclut en appliquant le théorème 1 .

Preuve de l'assertion. Cette preuve reprend des arguments de [Ke] et nous n'en donnerons que les grandes lignes. Compte tenu de l'écriture de $X$ dans des coordonnées adaptées on peut écrire (modulo un changement de temps):

$$
X^{*}=(a(\underline{x})+A(\underline{x}, r, z)) \frac{\partial}{\partial \underline{x}}+(b(\underline{x}) r+r B(\underline{x}, r, z)) \frac{\partial}{\partial r}-z^{q+1} \frac{\partial}{\partial z}
$$

où les restrictions de $A, B$ à $S^{1} \times\{0\} \times\{0\}$ sont nulles et $b(\underline{x})<b_{1}<0$ si $\underline{x} \in S^{1}$. Notons que $D_{\varepsilon}^{*}=D^{*} \cap C_{\varepsilon}^{*}$ est positivement invariant par $X^{*}$. Plus précisément toute demi-courbe intégrale $\sigma$ de $X^{*}$ issue d'un point de $D_{\varepsilon}^{*}$ est définie sur $[0, \infty[$ et $\omega(\sigma)$ est contenu dans $D^{*} \cap\{z=0\}$. Soient $\gamma^{*}, \sigma$ des demi-courbes intégrales de $X^{*}$ d'origines respectives $\left(\underline{x}_{0}, r_{0}, z_{0}\right),\left(\underline{x}_{1}, 0, z_{0}\right)$. Alors $v(t)=\gamma^{*}(t)-\sigma(t)=$ $(\underline{x}(t), p(t), 0), t>0$, est solution de l'équation différentielle $E$ sur $S^{1} \times[0,1[$, dépendant du temps obtenue en écrivant $v^{\prime}(t)=X^{*}\left(\gamma^{*}(t)\right)-X^{*}(\sigma(t))$. En écrivant 
que $\frac{d}{d t} \underline{x}(t)<\frac{1}{2} b_{1} \underline{x}(t)$, on peut utiliser la méthode classique d'approximations successives pour obtenir $v(t)$ comme point fixe de l'opérateur intégro-différentiel déduit de $E$. L'existence de la solution $\sigma: t \mapsto \sigma(t)$ cherchée et de la majoration associée s'en déduisent.

3. Fin de la preuve du théorème 2. Dans tout ce paragraphe, $\Gamma$ désigne un axe de spiralement en $p$, non dégénéré, pour une demi-courbe intégrale $\gamma_{0}$ de $X$. Nous prouverons le théorème en nous ramenant au cas réduit tout d'abord pour une singularité élémentaire et ensuite dans le cas général en effectuant des éclatements de points, de courbes et, éventuellement, une ramification. Nous utiliserons le résultat du paragraphe précédent sous la forme suivante. Si $(x, y, z)$ sont des coordonnées adaptées à une singularité réduite spiralante $(X, p)$ il existe $\varepsilon>0$ tel que $C_{\varepsilon}=\left\{x^{2}+y^{2}<1,0 \leq z<\varepsilon\right\}$ soit un domaine de tourbillonnement. De plus $X$ est transverse aux niveaux de $x^{2}+y^{2}$ sur $C_{\varepsilon} \backslash \Gamma$.

Proposition 3.1. Soit $(X, p)$ une singularité élémentaire qui possède un axe de spiralement $\Gamma$ non dégénéré (pour une courbe intégrale $\gamma_{0}$ ) transverse à une hypersurface lisse $D$ et soit $\left(\left(x_{0}, y_{0}, z_{0}\right), U\right)$ une carte affine en $p$ telle que $D=$ $\left\{z_{0}=0\right\}$ et $\Gamma \subset\left\{z_{0}>0\right\}$. Alors $\Gamma$ est un axe de tourbillonnement de $X$ qui possède un domaine de tourbillonnement $V$ semi algébrique dans les coordonnées $\left(x_{0}, y_{0}, z_{0}\right)$.

Preuve. D'après la proposition du paragraphe 1 , l'axe $\Gamma$ est lisse et $\mu(\Gamma)=0$. Il existe un changement de coordonnées du type $x=x_{0}+\varphi(z), y=y_{0}+\psi(z), z=z_{0}$ tel que $\Gamma=\{x=y=0, z>0\}$. Soit $q+1$ l'ordre en $z$ de la restriction de $X$ à $\Gamma$. On a

$$
X=a(x, y, z) \frac{\partial}{\partial x}+b(x, y, z) \frac{\partial}{\partial y}+\left(-z^{q+1}+c(x, y, z)\right) \frac{\partial}{\partial z}
$$

où $a(0,0, z)=b(0,0, z)=0$, ordre $c(0,0, z)>q+1$ et $D X(0)$ n'est pas nilpotente. Si $T I(\Gamma)=\left\{p_{i}\right\}$, notons $\pi$ le composé des $q+1$ éclatements de centres $p, p_{1} \cdots, p_{q}$ et soit $\left(\left(x^{\prime}, y^{\prime}, z\right), U^{\prime}\right)$ une carte affine en $p_{q}$ telle que $x^{\prime}=x z^{q+1}, y^{\prime}=y z^{q+1}$. Dans ces coordonnées le relevé $X^{\prime}$ de $X$ s'écrit

$$
X^{\prime}=\sum_{i=0}^{q} z^{i} L_{i}+z^{q+1}\left(h \frac{\partial}{\partial z}+\tilde{X}^{\prime}\right)
$$

où les $L_{i}$ et $\widetilde{X}^{\prime}$ sont comme dans la définition des coordonnées adaptées au cas réduit spiralant et $h(0)<0$. Ainsi $\left(x^{\prime}, y^{\prime}, z\right)$ sont des coordonnées adaptées au champ $h^{-1} X^{\prime}$. Il existe $\varepsilon>0$ tel que $C_{\varepsilon}=\left\{x^{\prime 2}+y^{\prime 2}<1,0<z<\varepsilon\right\}$ soit un domaine de tourbillonnement de $X^{\prime}$. De plus $X^{\prime}$ est transverse aux cylindres $\left\{x^{\prime 2}+y^{\prime 2}=r^{2},|z|<\varepsilon\right\}$ pour $r \leq 1$. Si $\alpha(z), \beta(z)$ sont des fonctions qui ont un ordre assez élevé en $z$, il existe $\varepsilon^{\prime}$ tel que

$$
C_{\varepsilon^{\prime}}(\alpha, \beta)=\left\{\left(x^{\prime}+\alpha(z)\right)^{2}+\left(y^{\prime}+\beta(z)\right)^{2}<1, \quad 0<z<\varepsilon^{\prime}\right\}
$$


soit un domaine de tourbillonnement de $\left(X^{\prime}, p^{\prime}\right)$. Sa projection

$$
\pi\left(C_{\varepsilon}^{\prime}(\alpha, \beta)\right)=\left\{\left(x+z^{q+1} \alpha(z)\right)^{2}+\left(y+z^{q+1} \beta(z)\right)^{2}<z^{q+1}, \quad 0<z<\varepsilon^{\prime}\right\}
$$

est un domaine de tourbillonnement de $(X, p)$. Ecrivons $\varphi(z)=\varphi_{s}(z)+f_{s}(z)$, $\psi(z)=\psi_{s}(z)+g_{s}(z)$ où $\varphi_{s}, \psi_{s}$ sont les jets d'ordre $s$ de $\varphi, \psi$. Pour $s$ assez grand

$$
V=\left\{\left(x-f_{s}(z)\right)^{2}+\left(y-g_{s}(z)\right)^{2}<z^{q+1}, 0<z<\varepsilon^{\prime}\right\}
$$

est un domaine de tourbillonnement de $(X, p)$ qui est semi algébrique dans les coordonnées initiales $\left(x_{0}, y_{0}, z_{0}\right)$ puisque $x-f_{s}(z)=x_{0}+\varphi_{s}(z), y-g_{s}(z)=$ $y_{0}+\psi_{s}(z)$.

Proposition 3.2. Supposons que $\Gamma$ soit un axe de spiralement non dégénéré en $p$ pour la demi-courbe intégrale $\gamma_{0}$ de X. Notons $T I(\Gamma)=\left\{p_{i}\right\}, \pi_{i}$ l'éclatement de centre $p_{i-1}, \Gamma_{i}=\pi_{i}^{-1}\left(\Gamma_{i-1}\right)$, et $((x, y, z), U)$ une carte affine en $p$. Il existe $k \geq 0$ et des coordonnées $\left(x_{k}, y_{k}, z_{k}\right)$ en $p_{k}$ telles que le relevé de $X$ en $p_{k}$ s'écrit $X_{k}=z_{k}^{s_{k}} X_{k}^{\prime}$ avec $D X_{k}^{\prime}\left(p_{k}\right) \not \equiv 0$ et $\Gamma_{k} \subset\left\{z_{k}>0\right\}$ est lisse transverse à $\left\{z_{k}=0\right\}$. De plus $\pi_{1} \circ \pi_{2} \cdots \circ \pi_{k}$ s'écrit algébriquement dans les coordonnées $(x, y, z),\left(x_{k}\right.$, $\left.y_{k}, z_{k}\right)$.

Preuve. Pour $i$ assez grand, $\Gamma_{i}$ est lisse, transverse au diviseur exceptionnel (qui est lisse en $p_{i}$ ). Sa tangente est une direction propre de $D X\left(p_{i}\right)$ associée à une valeur propre $\mu\left(\Gamma_{i}\right)=0$. On peut (modulo ces $i$ premiers éclatements) choisir des coordonnées $\left(x^{\prime}, y^{\prime}, z^{\prime}\right)$ telles que $\Gamma=\left\{x^{\prime}=y^{\prime}=0, z^{\prime}>0\right\}$, i.e que

$$
X=a \frac{\partial}{\partial x^{\prime}}+b \frac{\partial}{\partial y^{\prime}}+c \frac{\partial}{\partial z^{\prime}} \text { avec } a\left(0,0, z^{\prime}\right)=b\left(0,0, z^{\prime}\right) \equiv 0
$$

et $c\left(0,0, z^{\prime}\right)$ est d'ordre $d>1$ en $z^{\prime}$. Soient $\left(x_{1}^{\prime}=x / z^{\prime}, y_{1}=y / z^{\prime}, z_{1}^{\prime}=z^{\prime}\right)$ des coordonnées en $p_{1}$. Alors si $D X(p) \equiv 0$ il existe $s_{1}>0$ tel que $X_{1}=z^{s_{1}} X_{1}^{\prime}$ où

$$
X_{1}^{\prime}=a_{1} \frac{\partial}{\partial x_{1}^{\prime}}+b_{1} \frac{\partial}{\partial y_{1}^{\prime}}+c_{1} \frac{\partial}{\partial z_{1}^{\prime}} \text { avec } a_{1}\left(0,0, z_{1}^{\prime}\right)=b_{1}\left(0,0, z_{1}^{\prime}\right)=0
$$

et $c\left(0,0, z_{1}^{\prime}\right)$ est d'ordre $d_{1}<d$. Ainsi, il existe $k \geq 0$ et des coordonnées $\left(x_{k}^{\prime}, y_{k}^{\prime}, z_{k}^{\prime}\right)$ en $p_{k}$ telles que les conclusions de la proposition soient vérifiées. La carte affine $((x, y, z), U)$ étant fixée, on peut évidemment choisir une carte affine $\left(\left(x_{k}, y_{k}, z_{k}\right)\right)$, $U_{k}$ ) en $p_{k}$ telle que $\pi_{1} \circ \pi_{2} \circ \cdots \circ \pi_{k}$ soit algébrique et le diviseur exceptionnel soit $z_{k}=0$.

Si $D X_{k}^{\prime}\left(p_{k}\right)$ n'est pas nilpotente, il existe, d'après la proposition 3.1 un domaine de tourbillonnement $V_{k}$ de $\Gamma_{k}$ pour $\left(X_{k}, p_{k}\right)$ semi algébrique dans la carte $\left(\left(x_{k}, y_{k}\right.\right.$, $\left.\left.z_{k}\right), U_{k}\right)$. D'après le théorème de Tarski sa projection par $\pi_{1} \circ \pi_{2} \circ \cdots \pi_{k}$ est semi 
algébrique dans les coordonnées $(x, y, z)$. C'est un domaine de tourbillonnement d'axe $\Gamma$ pour $(X, p)$.

Si $D X_{k}^{\prime}\left(p_{k}\right)$ est nilpotente, compte tenu de l'algébricité de $\pi_{1} \circ \pi_{2} \circ \cdots \circ \pi_{k}$, la proposition suivante achève clairement la preuve du théorème 2 .

Proposition 3.3. Supposons que $D X(p) \not \equiv 0$ soit nilpotente, que $\Gamma$ soit un axe de spiralement en $p$ non dégénéré, lisse, pour une demi-courbe intégrale $\gamma_{0}$ de $X$ et que $\left(\left(x_{0}, y_{0}, z_{0}\right), U_{0}\right)$ soit une carte affine en $p$ telle que $\Gamma \subset\left\{z_{0}>0\right\}$ soit transverse $\grave{a}\left\{z_{0}=0\right\}$. Alors,$\Gamma$ est un axe de tourbillonnement de $X$ qui possède un domaine de tourbillonnement semi algébrique dans les coordonnées $\left(x_{0}, y_{0}, z_{0}\right)$.

Preuve. Soient $(x, y, z)$ des coordonnées en $p$ telles que $z=z_{0}, x=x_{0}-\varphi(z)$, $y=y_{0}-\psi(z)$, où $\Gamma=\left\{x_{0}=\varphi(z), y_{0}=\psi(z), z>0\right\}$. Quitte à effectuer les éclatements de centres $p, p_{1}, \cdots p_{q} \in T I(\Gamma)$, on peut supposer que, dans ces coordonnées,

$$
X=y \frac{\partial}{\partial x}+\sum_{i=1}^{q} z^{i} L_{i}(x, y)+z^{q+1}\left(-\frac{\partial}{\partial z}+V\right)
$$

où $L_{i}(x, y)=\left(\alpha_{i} x+\beta_{i} y\right) \frac{\partial}{\partial x}+\left(\gamma_{i} x+\delta_{i} y\right) \frac{\partial}{\partial y}, V=a \frac{\partial}{\partial x}+b \frac{\partial}{\partial y}$ avec $a(0,0, z)=b(0,0, z) \equiv 0$ et $q>0$ puisque $\gamma_{0}$ a un contact plat avec $\Gamma$. Distinguons deux cas.

$\mathbf{1}^{\text {ér }}$ cas, $\gamma_{1}=0$. Soit $\pi: M^{\prime} \longrightarrow U_{0} \cong \mathbb{R}^{3}$ l'éclatement de centre la droite $\Delta=$ $\{y=z=0\}$. Notons que $\Delta$ a un sens intrinsèque, c'est le lieu singulier de $X$ dans le diviseur. En particulier c'est une courbe algébrique dans les coordonnées initiales. Soient $\Gamma^{\prime}=\pi^{-1}(\Gamma), \gamma_{0}^{\prime}=\pi^{-1} \circ \gamma_{0}$ et soient $\left(x^{\prime}=x, y^{\prime}=y / z, z^{\prime}=z\right)$ des coordonnées au point $p^{\prime}=\omega\left(\Gamma^{\prime}\right)$. Dans ces coordonnées si $X^{\prime}$ est le relevé de $X$ par $\pi$ on a

$$
X^{\prime \prime}=\left.\left(z^{\prime}\right)^{-1} X^{\prime}\right|_{z^{\prime}}=\sum_{i=0}^{q} z^{\prime i} L_{i}^{\prime}+z^{\prime q^{\prime}}\left(-\frac{\partial}{\partial z}+V^{\prime}\right)
$$

où $q^{\prime}=q-1$, les $L_{i}^{\prime}, V^{\prime}$ sont comme plus haut et

$$
L_{0}^{\prime}\left(x^{\prime}, y^{\prime}\right)=\left(\alpha_{1} x^{\prime}+y^{\prime}\right) \frac{\partial}{\partial x}+\left(\gamma_{2} x^{\prime}+\delta_{1} y^{\prime}\right) \frac{\partial}{\partial y} .
$$

Puisque $\Gamma^{\prime}$ est un axe de spiralement non dégénéré de la courbe intégrale $\gamma_{0}^{\prime}$ de $X^{\prime \prime}$ on a encore $q^{\prime}>1$. Si $D X^{\prime \prime}\left(p^{\prime}\right)=D L_{0}^{\prime}(0)$ n'est pas nilpotente, $\left(X^{\prime \prime}, p^{\prime}\right)$ est une singularité réduite spiralante et les coordonnées $\left(x^{\prime}, y^{\prime}, z^{\prime}\right)$ lui sont adaptées. D'après les propositions 2.1, 2.2 le cylindre $C_{\varepsilon}^{\prime}=\left\{x^{\prime 2}+y^{\prime 2}<1, \quad 0<z^{\prime}<\varepsilon\right\}$ est un domaine de tourbillonnement d'axe $\Gamma^{\prime}$ pour $\left(X^{\prime \prime}, p^{\prime}\right)$. Par le même argument que dans la preuve de la proposition 3.1 on montre l'existence d'un domaine de tourbillonnement $V^{\prime} \subset C_{\varepsilon}^{\prime}$ d'axe $\Gamma^{\prime}$ pour $\left(X^{\prime}, p^{\prime}\right)$ dont la projection par les morphismes d'éclatement effectués est un domaine de tourbillonnement d'axe $\Gamma$ 
pour $(X, p)$, semi algébrique dans les coordonnées $\left(x_{0}, y_{0}, z_{0}\right)$. Si $D X^{\prime \prime}\left(p^{\prime}\right)$ est nilpotente on conclut immédiatement en raisonnant par récurrence sur $q$ puisque $q^{\prime}=q-1>1$.

$2^{\text {ème }}$ cas, $\gamma_{1} \neq 0$. Soit $\pi^{\prime}$ le composé de la ramification $\left(x, y, z^{\prime}\right) \rightarrow\left(x, y, z^{\prime 2}\right)$ par l'éclatement de centre $\left\{y=z^{\prime}=0\right\}$. Posons $\Gamma^{\prime}=\pi^{\prime-1}(\Gamma), \gamma_{0}^{\prime}=\pi^{\prime-1} \circ \gamma_{0}$ et soient $\left(x^{\prime}=x, y^{\prime}=y / z^{\prime 2}, z^{\prime}\right)$ des coordonnées en $p^{\prime}=\omega\left(\Gamma^{\prime}\right)$. Si $X^{\prime}$ est le relevé de $X$ par $\pi^{\prime}$ on a

$$
X^{\prime \prime}=\left(z^{\prime-1}\right) X^{\prime}=\sum_{i=0}^{q} z^{i} L_{i}+z^{\prime 2 q}\left(-1 / 2 \frac{\partial}{\partial z^{\prime}}+V^{\prime}\right)
$$

avec $L_{0}^{\prime}=y^{\prime} \frac{\partial}{\partial x^{\prime}}+\gamma_{1} x^{\prime} \frac{\partial}{\partial y^{\prime}}$. Puisque $D X^{\prime \prime}\left(p^{\prime}\right)=D L_{0}^{\prime}(0)$ n'est pas nilpotente, $\left(X^{\prime \prime}, p^{\prime}\right)$ est une singularité réduite spiralante. On conclut alors comme dans le cas $\gamma_{1}=0$.

\section{Preuve du corollaire de finitude}

Soit $p$ un point singulier de $X$. Nous pouvons clairement supposer que dim Sing $X \leq 1$. Ainsi Sing $X \backslash\{p\}$ est une union finie de demi-branches analytiques. L'ensemble des axes de spiralement de $X$ en $p$ contenus dans Sing $X$ est fini. Pour montrer que l'ensemble des axes de tourbillonnement non dégénérés est localement fini nous utiliserons un résultat "d'uniformisation locale" des champs vecteurs en dimension trois démontré dans [Ca]. Le résultat s'énonce en termes d'existence d'une stratégie gagnante pour un jeu à la Hironaka $\left[\mathrm{Hi}_{2}\right]$ que nous rappelons.

Soit $X$ un germe en $p$ de champ de vecteurs analytique sur une variété de dimension trois. Deux joueurs $A, B$ jouent au jeu suivant qui comporte deux étapes:

1) Le joueur $A$ choisit un centre d'éclatement $Y$ qui est soit le point $p$ soit une (un germe de) courbe lisse en $p$. On fait l'éclatement $\pi_{1}: M_{1} \rightarrow M_{0}=M$ et on considère $X_{1}$ le transformé strict de $X$ (relevé divisé).

2) Le joueur $B$ choisit un point $p_{1} \in \pi^{-1}(p)$ tel que $p_{1}$ n'est pas une singularité élémentaire de $X_{1}$. S'il ne peut pas choisir un tel $p_{1}$, le jeu s'arrête et le "joueur A a gagné". Sinon on germifie la situation en $p_{1}$ et le jeu recommence avec $X_{1}$.

Une "stratégie gagnante" pour $A$ est un critère de choix des centres d'éclatement tel que, toute réalisation du jeu (partie) respectant ce critère soit finie. Le résultat d'uniformisation s'énonce alors : Il existe une stratégie gagnante pour $A$ et les longueurs de toutes les réalisations possibles (à partir de $(X, p)$ ) sont uniformément bornées.

Prouvons le corollaire en raisonnant par l'absurde. Supposons qu'il existe une suite infinie $\left\{\Gamma_{n}\right\}$ d'axes de tourbillonnement non dégénérés pour $X$ tels que la suite $\left\{q_{n}\right\}=\left\{\omega\left(\Gamma_{n}\right)\right\}$ converge vers $p$ et $\Gamma_{n} \neq \operatorname{Sing} \Gamma_{n^{\prime}}$ si $n \neq n^{\prime}$. Initions le jeu d'Hironaka avec le choix de $Y$. Notons que les $\left(\Gamma_{n}, q_{n}\right)$ et $\left(Y, q_{n}\right)$ sont distincts pour $n$ assez grand. En effet, si ce n'est pas le cas, pour $n$ assez grand on a 

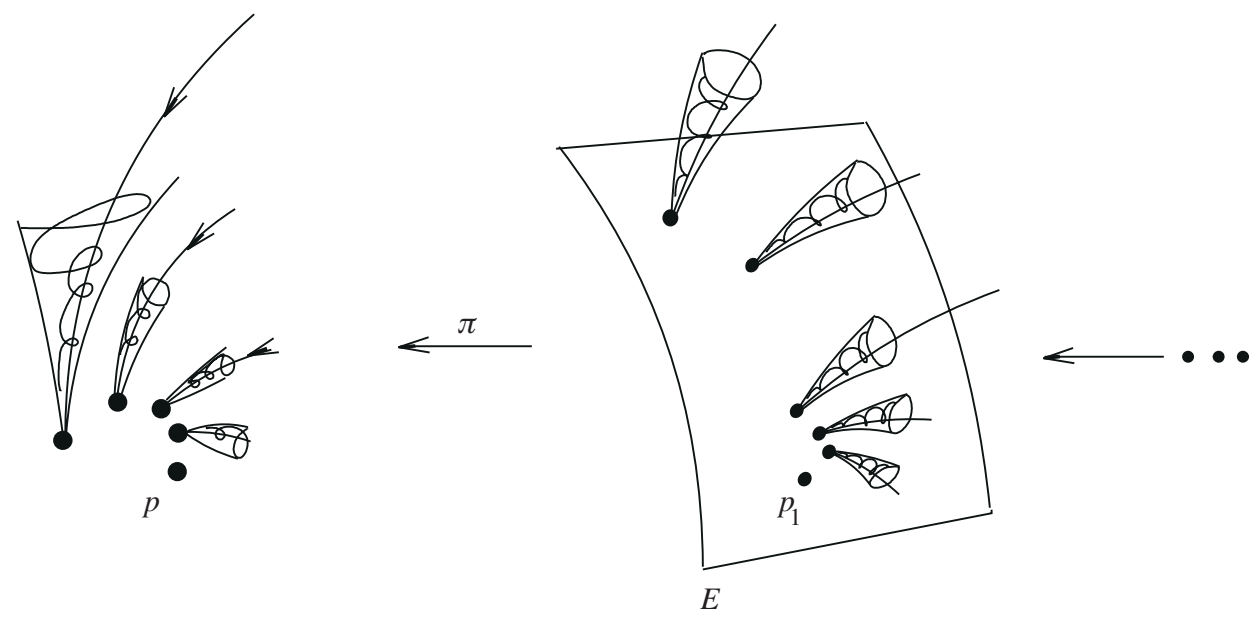

Figure 8 .

$q_{n} \neq q_{n^{\prime}}$ et puisque les $q_{n}$ appartiennent à $\operatorname{Sing} X \cap Y$, on a $Y \subset \operatorname{Sing} X$. Ce qui contredit l'hypothèse $\Gamma_{n} \not \subset$ Sing $X$. Soit alors $\pi_{1}: M_{1} \longrightarrow M$ l'éclatement de centre $Y$. Notons $\Gamma_{n, 1}$ le transformé strict de $\Gamma_{n}$ et $q_{n, 1}=\omega\left(\Gamma_{n, 1}\right)$. Puisque $\pi_{1}$ est un morphisme propre, quitte à choisir une sous suite des $\left\{\Gamma_{n}\right\}$, on peut supposer que $\left\{q_{n, 1}\right\}$ converge vers un point $p_{1} \in \pi^{-1}(p)$. Le joueur $B$ choisit le point $p_{1}$. En répétant ce type de choix pour le joueur $B$ un nombre fini de fois on obtient une singularité élémentaire $p_{k}$ qui ne vérifie pas la conclusion du corollaire du paragraphe II.

\section{Coordonnées adaptées et équilibrées}

Soit $\Gamma$ un axe de tourbillonnement non dégénéré, lisse de $X$ en un point $p$. Le but de ce dernier paragraphe est de montrer que la dynamique de $X$ induite sur un domaine de tourbillonnement bien choisi $V$ de $\Gamma$ est équilibrée au sens suivant: il existe une carte affine $((x, y, z), U)$ en $p$ telle que l'on ait:

$$
\Gamma=\{x=y=0, \quad z>0\}, \quad V=\left\{x^{2}+y^{2}<z^{n}, \quad 0<z<\varepsilon\right\}
$$

et telle que $X$ soit transverse aux niveaux de $x^{2}+y^{2}$, z et $y / x$ sur $V \backslash \Gamma$. En particulier la "rotation autour de $\Gamma$ " serait strictement monotone autour de $\Gamma$.

Proposition. Si $(X, p)$ est une singularité réduite spiralante, il existe des coordonnées $(x, y, z)$ adaptées à $(X, p)$ telles que $\Gamma=\{x=y=0, z>0\}$ soit un axe de tourbillonnement de domaine de tourbillonnement $C_{\varepsilon}$ et telles que $X$ soit transverse aux niveaux de $x^{2}+y^{2}$, z et $y / x$ sur $C_{\varepsilon} \backslash \Gamma$.

D'après les propositions 2.1, 2.2, nous devons seulement prouver que nous pouvons choisir des coordonnées adaptées $(x, y, z)$ telles que $X$ soit transverse au feuilletage 

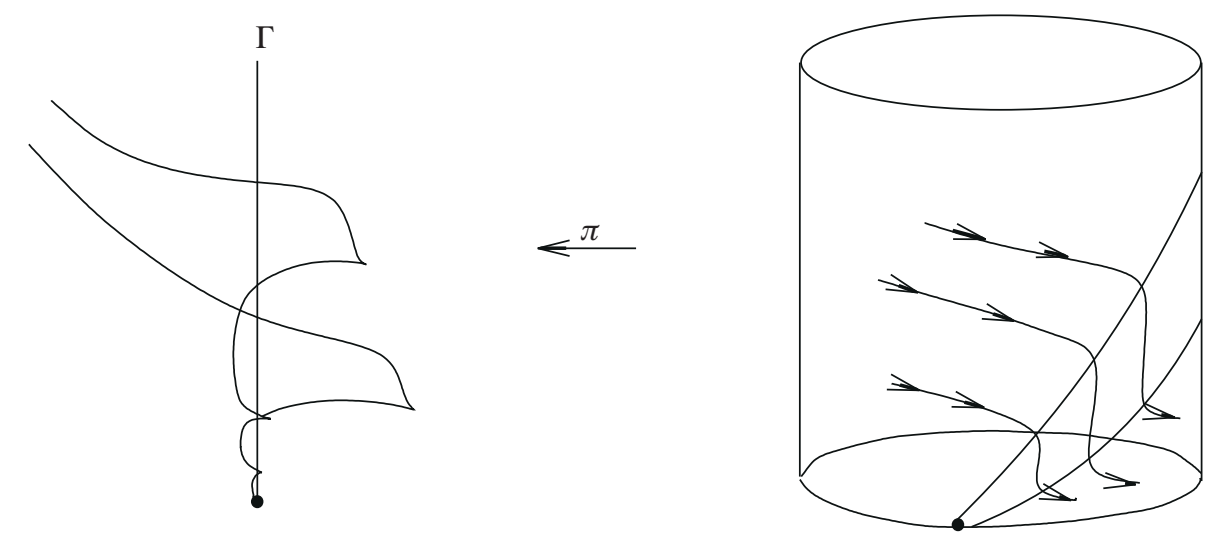

Figure 9 .

$y d x-x d y=0$ dans le cas non asymptotiquement monodromique. Avant d'aborder cette preuve, examinons tout d'abord l'exemple suivant:

$$
X=-\left(x \frac{\partial}{\partial x}+y \frac{\partial}{\partial y}\right)+y \frac{\partial}{\partial x}-\left(3 y z+2 x z^{2}\right) \frac{\partial}{\partial y}-z^{2} \frac{\partial}{\partial z} .
$$

Notons tout d'abord que $X$ n'est pas transverse au feuilletage $x d y-y d x=0$. En effet, la fonction $(x d y-y d x)(X)$ s'annule sur les surfaces $y=-x z, y=-2 x z$. Montrons que $\Gamma=\{x=y=0, z>0\}$ est un axe de tourbillonnement. Soient $\pi$ l'éclatement de centre $\{x=y=0\}$ et $D$ son diviseur exceptionnel. Le relevé $X^{\prime}$ de $X$ possède un seul point singulier $p^{\prime} \in \pi^{-1}(0)$. Soient $x^{\prime}=x, y^{\prime}=y / x, z^{\prime}=z$ des coordonnées centrées en ce point. On a

$$
Y=\left.X^{\prime}\right|_{D}=-\left(y^{\prime 2}+3 z^{\prime} y^{\prime}+2 z^{\prime 2}\right) \frac{\partial}{\partial y^{\prime}}-z^{\prime 2} \frac{\partial}{\partial z^{\prime}} .
$$

D'après la preuve de la proposition 2.2 il suffit de montrer qu'il n'existe pas de courbe $\sigma$ de $\left.Y\right|_{\{z>0\}}$ telle que $\omega(\sigma)=\left\{p^{\prime}\right\}$. Or $\{z=0\}$ est la seule direction réelle du cône tangent à $Y$ en $p^{\prime}$ et en éclatant ce point on obtient le résultat cherché. Faisons le changement de coordonnées $y^{\prime *}=y^{\prime}+3 / 2 z^{\prime}$ qui correspond au changement $y^{*}=y+3 / 2 x z$ à l'origine de $\mathbb{R}^{3}$. Le champ $Y$ s'écrit alors

$$
Y=-\left\{y^{\prime * 2}+5 / 4 z^{\prime 2}\right\} \frac{\partial}{\partial y^{\prime *}}-z^{\prime 2} \frac{\partial}{\partial z^{\prime}} .
$$

Il est transverse aux niveaux de $y^{\prime *}=$ cste lorsque $z^{\prime} \neq 0$. On en déduit que $X$ est transverse au feuilletage $y^{*} d x-x d y^{*}=0$.

Preuve de la proposition. D'après la définition des coordonnées adaptées on a

$$
X=\sum_{i=0}^{\infty} z^{i} L_{i}+z^{q+1}\left(-\frac{\partial}{\partial z}+\widetilde{X}\right), \quad q>1 \text { et } \nu_{x, y}(\widetilde{X}) \geq 2 .
$$


De plus $X$ n'étant pas asymptotiquement monodromique on a $L_{0}=-R$ ou $L_{0}=$ $-R+y \frac{\partial}{\partial x}$ avec $R=x \frac{\partial}{\partial x}+y \frac{\partial}{\partial y}$. Notons comme dans l'exemple ci-dessus $\pi$ l'éclatement de centre $\{x=y=0\}, D$ le diviseur exceptionnel et $Y=\left.X^{\prime}\right|_{D}$. Puisque $\Gamma=\{x=y=0, z>0\}$ est un axe de tourbillonnement de $X$ on sait d'après la preuve de la proposition 2.2 que:

(*) Si $\sigma$ est une courbe intégrale de $Y$ avec $\sigma(0) \in \pi^{-1}\{z>0\}$, on a $\omega(\sigma)=$ $\pi^{-1}(0)=F \simeq S^{1}$.

(**) Les singularités simples obtenues par réduction des singularités du feuilletage $\mathcal{L}$ défini par $Y$ sur $D$ sont des "coins" (points de croisement du diviseur exceptionnel) ou des points du transformé strict de $F$.

Notons $t$ le premier indice $i$ tel que $L_{t} \neq \alpha_{t} R$. Si $t>q$, la fibre $F$ n'est pas invariante par $\mathcal{L}$, ce qui contredit $(*)$. Si $t=q$, le feuilletage $\mathcal{L}$ possède une singularité simple sur $F$, ce qui contredit encore $(*)$. Posons

$$
\widetilde{L}_{i}=L_{i}-\alpha_{i} R=\beta_{i} y \frac{\partial}{\partial x}+\left(\gamma_{i} x+\widetilde{\delta}_{i} y\right) \frac{\partial}{\partial y} .
$$

On peut supposer que $L_{t}$ est écrite sous forme de Jordan. C'est-à-dire que l'on a: soit $\widetilde{L}_{t}=\widetilde{\delta}_{t} y \frac{\partial}{\partial y}$ avec $\widetilde{\delta}_{t} \neq 0$, soit $\widetilde{L}_{t}=\beta_{t}\left(y \frac{\partial}{\partial x}-x \frac{\partial}{\partial y}\right)$ avec $\beta_{t} \neq 0$, soit $\widetilde{L}_{t}=y \frac{\partial}{\partial x}$. Le premier cas contredit $(*)$ car $\mathcal{L}$ a une singularité simple sur $F$. Dans le second cas, $\mathcal{L}$ n'a pas de singularité sur $F$ et il est clair que $X$ est transverse au feuilletage $y d x-y d x=0$. Etudions le troisième cas, $\widetilde{L}_{t}=y \frac{\partial}{\partial x}$. Dans les coordonnées $\left(x, y^{\prime}=y / x, z\right)$ le champ $Y$ s'écrit:

$$
\begin{gathered}
Y=z^{t}\left\{\left(a(z) y^{\prime 2}+b(z) y^{\prime}+c(z)\right) \partial / \partial y^{\prime}-z^{q^{\prime}} z \partial / \partial z\right\}, \quad q^{\prime}=q-t \\
a(z)=-1-\sum_{i=1}^{\infty} \beta_{t+i} z^{i}, \quad b(z)=\sum_{i=1}^{\infty} \widetilde{\delta}_{t+i} z^{i}, \quad c(z)=\sum_{i=1}^{\infty} \gamma_{t+i} z^{i} .
\end{gathered}
$$

Soit $N\left(y^{\prime}\right)$ le nuage de points de $Y\left(y^{\prime}\right) / z^{t}$ et $\Delta\left(y^{\prime}\right)$ le polygône de Newton de $N\left(y^{\prime}\right) \cup\left\{\left(q^{\prime}, 1\right)\right\}$. Le premier sommet de $\Delta\left(y^{\prime}\right)$ est $(0,2)$ et si $-1 / d\left(y^{\prime}\right)$ est sa pente, alors $d\left(y^{\prime}\right) \leq q^{\prime}$. En suivant une méthode de préparation inspirée d'Hironaka $\left[\mathrm{Hi}_{2}\right]$, nous dirons que la coordonnée $y^{\prime}$ est équilibrée si l'une des propriétés suivantes arrive:

i) Le deuxième sommet du premier côté est $\left(d\left(y^{\prime}\right), 1\right)$ ou $d\left(y^{\prime}\right) \notin \mathbb{Z}$.

ii) La pente $d=d\left(y^{\prime}\right) \in \mathbb{Z}$, le deuxième sommet est $(2 d, 0)$, le point $(d, 1) \notin \mathbb{N}\left(y^{\prime}\right)$ et il n'existe pas $\lambda \in \mathbb{R}$ tel que si $y^{\prime *}=y^{\prime}+\lambda z^{d}$, alors soit $d\left(y^{\prime *}\right)>d\left(y^{\prime}\right)$, soit la longueur du premier segment decroît strictement.

Le changement $y^{\prime} \longmapsto y^{\prime *}$ correspond à $y \longmapsto y+\lambda x z^{d}$ qui est algébrique et conserve le caractère adapté des coordonnées. Après un nombre fini de changements de ce type, on peut suposer que $y^{\prime}$ est équilibrée. Montrons que les coordonnées 
choisies sont déjà bonnes. Elles possèdent déjà la propriété suivante de contact maximal.

"Soit $p^{\prime}=p_{0}$ l'origine de la carte $\left(y^{\prime}, z\right)$ de $D$, alors $p_{0} \in$ Sing $\mathcal{L}$ et $d_{0}=$ $d\left(y^{\prime}\right) \geq 1$. Eclatons $p_{0}$ et soit $p_{1}$ le point qui correspond au transformé strict de $y^{\prime}=0$. On a des coordonnées $\left(y_{1}^{\prime}=y^{\prime} / z, z\right)$ en $p_{1}$. Alors la coordonnée $y_{1}^{\prime}$ est équilibrée par rapport au transformé $Y_{1}$ de $Y$. De plus $d\left(y_{1}^{\prime}\right)=d\left(y^{\prime}\right)-1, q_{1}^{\prime}=q^{\prime}-1$ et le nouveau polygône $\Delta\left(y_{1}^{\prime}\right)$ s'obtient en appliquant la transformation linéaire $(u, v) \longmapsto(u+v-2, v)$ à $\Delta\left(y^{\prime}\right) "$.

En itérant cette procédure on voit que le cas i) n'arrive pas sinon on a une singularité simple sur le transformé strict d'une courbe du type $y^{\prime 2}-z^{2 d+1}=0$ ou sur le transformé strict de $y^{\prime}=0$, ce qui contredit $(* *)$. Etudions le cas ii) en posant $f(x, y, z)=x X(y)-y X(x)$,

$$
f(x, y, z)=\left\{a(z) y^{2}+b(z) y x+c(z) x^{2}+z^{q^{\prime}+1}(x \widetilde{X}(y)-y \widetilde{X}(x))\right\} z^{t} .
$$

Comme l'ordre de $x \widetilde{X}(y)-y \widetilde{X}(x)$ est $\geq 3$ en $x, y$, pour montrer que $f(x, y, z)$ garde un signe constant sur la région qui nous intéresse, il suffit de montrer que le discriminant $A(z)=b(z)^{2}-4 a(z) c(z)$ est strictement négatif pour $0<z<<1$. Comme $y^{\prime}$ est equilibrée, on sait que $\nu(b(z))>d$ et que $A(z)=\gamma z^{2 d}+z^{2 d+1}(\ldots)$ où $\gamma<0$ si $d<q^{\prime}$ et $\gamma<-q^{\prime}$ si $d=q^{\prime}$. On a toujours $\gamma<0$ et $A(z)<0$ pour $0<z<<1$. Ceci termine la démonstration.

\section{Bibliographie}

[A-H-V] J.-M. Aroca, A. Hironaka and J.-L. Vicente, Introduction to the Theory of Infinitely Near Singular Points. Mem. Math. Inst. Jorge Juan 28 Madrid CSIC (1977).

[Bo] E. Borel, Mémoire sur les Séries Divergentes. Ann. Sci. de l'E.N.S., $3^{\text {ème }}$ série, t. 16 (1899), 521-568.

[Bon-Du] P. Bonckaert and F. Dumortier, Smooth invariant curves for germs of vector fields in $\mathbb{R}^{3}$ whose linear part generates rotations. Journ. Diff. Eq. 62 (1986), 95-116.

[Br] M. Brunella, Instability of Equilibria in Dimension Three. Ann. Inst. Fourier 48(5) (1998), 1345-1357.

[Ca] F. Cano, Desingularization Strategies for a Three-dimensional Vector Field. Lect. Notes in Math. $\mathrm{n}^{\circ} 1259$. Springer-Verlag (1987).

[Ha] J. Hale, Ordinary Differential Equations. Pure and Applied Mathematics. XXI. Wiley-Interscience 1969.

[Hi $\left.{ }_{1}\right]$ H. Hironaka, Introduction to Real Analytic Sets and Real Analytic Maps. Instituto Matematico "L. Tonelli", Pisa 1973.

$\left[\mathrm{Hi}_{2}\right]$ H. Hironaka, Characteristic Polyhedra of Singularities. J. of Math. of Kyoto Univ., 7(3) (1968), 251-293.

[Hi-P-S] M. Hirsch, C. Pugh and M. Shub, Invariant Manifolds. Lect. Notes in Mathematics, 583. Springer Verlag (1977).

[Hu] Lin Hu Xing, Sur la structure des champs de gradients de fonctions analytiques réelles. Thèse Paris VII (1992).

[Ke] A. Kelley, Stability of the Center-Stable Manifold. J. Math. Anal. Appl. 18 (1967), 336-344. 
[Kh] A. Khovanskii, Real analytic Varieties with the Finiteness Property and Complex Abelian Integrals. Funct. Anal. And Appl. 18 119-127.

[Lo1] S. Lojasiewicz, Une propriété topologique des sous-ensembles analytiques réels. Colloques Internationaux du C.N.R.S. $\mathrm{n}^{\circ} 117$, les équations aux dérivées partielles, Paris 25-30 juin (1962) 87-89.

$\left[\mathrm{LO}_{2}\right]$ S. Lojasiewicz, Ensembles semi-analytiques. Séminaire E.C. Zeeman, Seminar on Combinatorial Topology I.H.E.S. p.92.

[Ly] A. M. Lyapunov, Stability of Motion. Math. in Science and Engineering, 30, Academic Press (1966).

[Me-Pa] J. de Melo and J. Palis, Geometric theory of dynamical systems. Springer-Verlag (1982).

[No-Ya] D. Novikov and S. Yakovenko, Trajectories of polynomial vector fields and ascending chains of polynomial ideals. Ann. Inst. Fourier 49 (1999), 563-609.

[Po] H. Poincaré, Mémoire sur les courbes définies par une équation différentielle. $J$. de Math. 7 (3) (1881).

[Sa] F. Sanz, Sur les trajectoires d'un champ de gradient. Ann. Inst. Fourier 48 (4) (1998), 1045-1067.

[Se] A. Seidenberg, Reduction of Singularities of the differentiable equation $A d Y=$ BdX. Am. J. of Math. (1968), 248-269.

[Ta] A. Tarski, A Decision Method For Elementary Algebra and Geometry. Berkeley and Los Angeles (1951).

[Tak] F. Takens, Singularities of Vector Fields. Publ. Math. IHES 43 (1974), 48-100.

$\left[\mathrm{Th}_{1}\right]$ R. Thom, Gradients of analytic functions. Proceedings of Seventh National Mathematics Conferences p. 364-371 Azarabadegan Univ. Tabriz 1977 (D.R.J. Chillingworth).

[Th 2$]$ R. Thom, Limit set of leaves of analytic foliations. Notes manuscrites (1980).

\section{F. Cano}

Dpto. Algebra, geometria y topologia

Facultad de Ciencias

E-47005 Valladolid

Spain

e-mail: fcano@cpd.uva.es

R. Moussu

Université de Bourgogne

Lab. Topologie, CNRS UMR 5584

Dép. Mathématiques

B. P. 400

F-21011-Dijon Cedex

France

e-mail: rmoussu@u-bourgogne.fr
F. Sanz

Dpto. Algebra, geometria y topologia

Facultad de Ciencias

E-47005 Valladolid

Spain

e-mail: fsanz@agt.uva.es

(Received: December 12, 1998) 